\title{
Genetic Analysis of Right Heart Structure and Function in 40,000 People
}

James P. Pirruccello ${ }^{\star 1,2,3,4}$, Paolo Di Achille*3,5 , Victor Nauffal ${ }^{\star 3,6}$, Mahan Nekoui ${ }^{3,4}$, Samuel N. Friedman ${ }^{3,5}$, Marcus D. R. Klarqvist ${ }^{3,5}$, Mark D. Chaffin ${ }^{3}$, Shaan Khurshid ${ }^{1,2,3}$, Carolina Roselli ${ }^{3,7}$, Puneet Batra ${ }^{5}$, Kenney $\mathrm{Ng}^{8}$, Steven A. Lubitz, ${ }^{1,2,3,4}$, Jennifer E. Ho ${ }^{1,2,4}$, Mark E. Lindsay ${ }^{1,2,3,4,9}$, Anthony A. Philippakis ${ }^{5,10}$, Patrick T. Ellinor ${ }^{1,2,3,4}$

1 Cardiology Division, Massachusetts General Hospital, Boston, Massachusetts, USA

2 Cardiovascular Research Center, Massachusetts General Hospital, Boston, Massachusetts, USA

3 Cardiovascular Disease Initiative, Broad Institute of MIT and Harvard, Cambridge, Massachusetts, USA

4 Harvard Medical School, Boston, Massachusetts, USA

5 Data Sciences Platform, Broad Institute of MIT and Harvard, Cambridge, Massachusetts, USA

6 Cardiovascular Division, Brigham and Women's Hospital, Boston, Massachusetts, USA

7 University Medical Center Groningen, University of Groningen, Groningen, NL

8 IBM Research, Cambridge, Massachusetts, USA

9 Thoracic Aortic Center, Massachusetts General Hospital, Boston, Massachusetts, USA

$10 \mathrm{GV}$, Mountain View, California, USA

* = These authors contributed equally to this work.

Running Title: Genetics of the right heart

Keywords: Right ventricle, right atrium, pulmonary artery, deep learning, Poisson surface reconstruction, cardiovascular disease, genetics

Corresponding Author:

Patrick T. Ellinor, MD, PhD

Cardiovascular Disease Initiative

The Broad Institute of MIT and Harvard

75 Ames Street

Cambridge, MA 02142

ellinor@mgh.harvard.edu 
The heart evolved hundreds of millions of years ago. During mammalian evolution, the cardiovascular system developed with complete separation between pulmonary and systemic circulations incorporated into a single pump with chambers dedicated to each circulation. A lower pressure right heart chamber supplies deoxygenated blood to the lungs, while a high pressure left heart chamber supplies oxygenated blood to the rest of the body. Due to the complexity of morphogenic cardiac looping and septation required to form these two chambers, congenital heart diseases often involve maldevelopment of the evolutionarily recent right heart chamber. Additionally, some diseases predominantly affect structures of the right heart, including arrhythmogenic right ventricular cardiomyopathy (ARVC) and pulmonary hypertension. To gain insight into right heart structure and function, we fine-tuned deep learning models to recognize the right atrium, the right ventricle, and the pulmonary artery, and then used those models to measure right heart structures in over 40,000 individuals from the UK Biobank with magnetic resonance imaging. We found associations between these measurements and clinical disease including pulmonary hypertension and dilated cardiomyopathy. We then conducted genome-wide association studies, identifying 104 distinct loci associated with at least one right heart measurement. Several of these loci were found near genes previously linked with congenital heart disease, such as NKX2-5, TBX3, WNT9B, and GATA4. We also observed interesting commonalities and differences in association patterns at genetic loci linked with both right and left ventricular measurements. Finally, we found that a polygenic predictor of right ventricular end systolic volume was associated with incident dilated cardiomyopathy (HR 1.28 per standard deviation; $\mathbf{P}=$ 2.4E-10), and remained a significant predictor of disease even after accounting for a left ventricular polygenic score. Harnessing deep learning to perform large-scale cardiac phenotyping, our results yield insights into the genetic and clinical determinants of right heart structure and function. 
The heart evolved hundreds of millions of years ago as a tubular organ ${ }^{1}$. Septation of the main pumping chamber of the heart into distinct left and right ventricles evolved later in birds, mammals, and some reptiles, and is under the control of conserved transcription factors such as $T B X 5^{2}$. Substantially greater delivery of oxygen to the systemic circulation-and to the heart itself-is the putative advantage of this separation of the circulatory system into a left heartdriven systemic circuit and a right heart-driven pulmonary circuit ${ }^{3}$.

The structures of the left and right heart are derived from different progenitor cell populations and operate under different pressure regimes: the left heart operates against high pressure, while the right heart generally faces little afterload. During embryogenesis, the left ventricle forms from the first heart field, while the right ventricle, the outflow tract, and portions of the atria form from the second heart field ${ }^{4-7}$. Septation of the outflow tract also requires neuroectodermal neural crest cells ${ }^{8-10}$.

The distinct embryological origins of the right and left ventricles likely explain, in part, the existence of right heart-predominant pathologies. These include arrhythmogenic right ventricular cardiomyopathy (ARVC) $)^{11-14}$, Brugada syndrome, and pulmonary hypertension. In addition, right ventricular dysfunction can play a role in other heart failure syndromes. The function of the right heart is an important determinant of outcomes in people who have heart failure with either reduced (HFrEF) or preserved left ventricular ejection fraction (HFpEF) $)^{15-17}$. HFpEF represents a heterogeneous set of diseases for which very few disease-modifying therapies exist. Consequently, there is substantial interest in identifying new therapies for conditions such as right ventricular dysfunction ${ }^{18-21}$.

The distinct pathologies, embryology, and physiology of the right heart motivated our efforts to quantify right heart structure and function, and to probe the common genetic basis for human variation in these measurements. 


\section{Results}

In this work, we developed deep learning models to determine the dimensions and function of the right atrium (RA), the right ventricle (RV), and the pulmonary artery (PA) in up to 45,000 UK Biobank participants. We then evaluated the epidemiologic associations, pathologic outcomes, and the common genetic basis of variation in these right heart structures.

\section{Reconstruction of right heart structures from cardiovascular magnetic resonance images}

We first derived right heart measurements in the UK Biobank imaging substudy of over 45,000 people ${ }^{22-24}$ using deep learning models. To do so, a cardiologist created training data for deep learning models by manually tracing the right atrium and right ventricle in the four-chamber long axis view, and the right ventricle and pulmonary artery in the short axis view (Figure 1). This process, called semantic segmentation, yielded anatomical labels identifying the pixels belonging to cardiac structures in 714 short axis images and 445 four-chamber long axis images. Two U-Net derived deep learning models, containing long-range skip connections that allow for pixel-accurate segmentation, were then trained from these data: one for the fourchamber long axis view and another for the short axis views ${ }^{25,26}$. The deep learning models were then used to produce pixel labels for the remainder of the images. Quality assessment is detailed in the Online Methods and Supplementary Note.

The deep learning model output was then post-processed to extract measurements of the right atrium, the right ventricle, and the pulmonary artery. The right atrium was only consistently visible in one view (the four-chamber long axis view), and therefore a 2-dimensional area was computed by summing the pixels and multiplying by their width and height. We computed the maximum and minimum area during the cardiac cycle, as well as the fractional area change (RA FAC), which is the ratio of the change in area between the maximum and minimum area divided by the maximum area.

The right ventricle has a complex 3-dimensional geometry; to estimate right ventricular structure, we integrated data from the short axis views and the four-chamber long axis view with a Poisson surface reconstruction approach, detailed in the Online Methods. We measured the maximum volume (right ventricular end diastolic volume; RVEDV), the minimum volume (right ventricular end systolic volume; RVESV), the difference between those two volumes (stroke volume), and the ejection fraction (RVEF).

The pulmonary trunk's elliptical minor axis (diameter) was computed from short axis images at end-systole. For participants whose pulmonary trunk was visible in multiple short-axis slices, we refer to the component closest to the right ventricle as the pulmonary root, and the distal-most component as the proximal pulmonary artery.

In total, we were able to measure at least one right heart structure in 45,456 individuals, of whom 41,101 contributed to at least one genome-wide association study after genotyping quality control and exclusion for prevalent disease (Table 1 and Supplementary Figure 1). 


\section{Right heart structures are correlated with their left-heart counterparts}

The mean and standard deviation of the right atrial area measurements, right ventricular volumes, and pulmonary artery diameters are described in Table 1 and visualized in

Supplementary Figure 2. Standard values aggregated by age bands and sex for each of the phenotypes are reported in Supplementary Table 1. The estimates of right atrial area from the four-chamber view are similar to those previously reported ${ }^{27}$, as are the proximal pulmonary artery diameters ${ }^{28}$. The estimates of right ventricular stroke volume are comparable to prior reports, but both end diastolic and end systolic volumes are approximately $10 \mathrm{~mL}$ greater than those previously reported for steady-state free precession magnetic resonance imaging ${ }^{29}$. Consequently, the right ventricular ejection fraction estimates are proportionally lower than those of Foppa, et al.

We incorporated previously reported left ventricular traits and aortic traits ${ }^{30,31}$ in order to analyze cross-correlation between phenotypes of the right and left heart structures(Supplementary Figure 3). The volumetric measurements of the right and left ventricles were well correlated with one another (correlation between ventricular volumes was 0.84 at end-diastole and 0.71 at endsystole). In contrast, there was poorer correlation between right and left ventricular ejection fraction (correlation 0.48 ). This is consistent with drivers of contractility being only partially shared between the two ventricles, as well as multiplicative error due to the calculation of ejection fraction from two separately measured volumes. The ventricles nevertheless had well correlated stroke volumes (correlation 0.80 ), which is expected because stroke volume at steady-state is expected to be equal for both ventricles in the absence of valvular regurgitation or shunt.

The proximal pulmonary artery diameter was modestly correlated with right ventricular end systolic volume (correlation 0.49), suggesting shared right-heart related influences on the pulmonary artery diameter and right ventricular volumes. In addition, the pulmonary artery diameter and that of the ascending aorta—which share an embryological origin-were modestly correlated (correlation 0.45).

\section{Right heart measurements are associated with cardiovascular diseases}

We tested PheCode-based disease definitions, which are derived from hospital diagnosis codes, for association with right heart phenotypes ${ }^{32}$. The right heart phenotypes were strongly correlated with atrial arrhythmias. The right atrial phenotypes were also associated with valvular diseases; the right ventricular phenotypes with obesity and heart failure; and the pulmonary artery phenotypes with obesity, blood pressure, and sleep disorders (Figure 2, Supplementary Table 2).

We also focused on three diseases with putative chamber-specific links to the right heart. We identified 1,033 individuals with a diagnosis of atrial fibrillation or flutter prior to undergoing MRI; 282 with congestive heart failure; and 21 with pulmonary hypertension (Supplementary Table 3). In a linear model, the right atrial FAC was 1.1 standard deviations (SD) lower among those with a history of atrial fibrillation or flutter than those without $(P=2.6 E-287)$. The RVEF was 0.51 
SD lower among those with heart failure $(P=6.6 \mathrm{E}-19)$. The proximal pulmonary artery diameter was $0.84 \mathrm{SD}$ larger among those with pulmonary hypertension $(\mathrm{P}=5.9 \mathrm{E}-05)$. These findings confirmed expected structural correlations with prevalent cardiovascular diseases.

For two cardiovascular diseases_-pulmonary hypertension and congestive heart failure - we modeled right ventricular volumes over the course of the cardiac cycle for individuals with and without disease (Figure 3). In these models, pulmonary hypertension (present in 21 participants) was associated with elevated volumes throughout the entire cardiac cycle, yielding a reduced RVEF. The excess volume that was attributable to disease accounted for as much as $34 \%$ of the total right ventricular volume $(P=1.8 \mathrm{E}-08)$ at end-systole and $15 \%(P=1.4 \mathrm{E}-04)$ at end-diastole. Congestive heart failure (present in 282 participants) was also associated with elevated end-systolic volumes ( $14 \%$ elevation; $P=6.0 \mathrm{E}-17$ ), but not with end-diastolic volumes ( $1 \%$ elevation; $P=0.44$ ). As a negative control, 3,949 participants with cataract-a disease of the lens of the eye that is not expected to be linked to right ventricular size-was associated with no significant difference in right ventricular volumes compared to cataract-free individuals. These results demonstrate that different cardiovascular diseases can yield distinct perturbations of right ventricular volumes, and highlight the significant impact of pulmonary hypertension on right ventricular structure throughout the cardiac cycle in this population.

\section{Right heart traits are heritable and genetically correlated with left heart traits}

We then conducted genetic analyses of the right heart phenotypes. The size-related phenotypes showed significant heritability using BOLT-REML (as high as 0.37 for the maximum right atrial volume, 0.4 for right ventricular end-diastolic volume, and 0.42 for the pulmonary artery root diameter $)^{33,34}$. Heritabilities were lower for measurements of right heart function, such as RVEF which had a heritability of 0.23 .

We assessed genetic correlation between the right heart structures and previously reported left heart structures that include the left ventricle and the ascending aorta. Using individual-level data with BOLT-REML ${ }^{34}$, we found strong genetic correlation between the right and left ventricles ( $r g=0.86$ between RVEDV and LVEDV; $r g=0.75$ between RVESV and LVESV; and $\mathrm{rg}=0.61$ between RVEF and LVEF). The proximal pulmonary artery diameter was most strongly correlated with the ascending aortic diameter $(\mathrm{rg}=0.60)$. The genetic correlation matrix across all of the derived cardiovascular traits is available in Supplementary Table 4 and Supplementary Figure 4, with trait heritabilities along the diagonal.

\section{Common genetic basis for the dimensions and function of the right heart}

After establishing the heritability of the right heart traits, we conducted genome-wide association studies (GWAS) of each trait. We excluded participants with diagnoses of heart failure, atrial fibrillation, or myocardial infarction prior to their magnetic resonance imaging study (Supplementary Figure 1). We conducted nine primary GWAS: maximum and minimum right atrial area; RA FAC; RVESV, RVEDV, RVSV, and RVEF; pulmonary artery root diameter; and proximal pulmonary artery diameter. Up to 40,466 participants were included in these analyses, 
and we tested 11.6 million imputed SNPs with minor allele frequency (MAF) > 0.005 (Table 2, Figure 4). In addition, we evaluated the body surface area (BSA)-indexed versions of all traits except for RA FAC and RVEF (which are dimensionless), leading to a total of 16 GWAS (Supplementary Table 5, Supplementary Figure 5). Allowing loci to be counted once per trait, we identified 243 trait-locus pairs at a commonly used significance threshold of 5E-08.

Accounting for multiple traits sharing loci, we identified 104 independent loci. Of these 104 loci, 66 were associated with at least two traits, and one locus (near WNT9B/GOSR2/MYL4) was associated with 12 right heart phenotypes. The greatest lambda GC was 1.20 from the BSAindexed pulmonary artery root GWAS; Idsc revealed an intercept of 1.04, consistent with polygenicity rather than inflation (Supplementary Table 6) ${ }^{35}$. Six lead SNPs had HardyWeinberg equilibrium (HWE) P < 1E-06; re-analysis of those SNPs in a strictly European subset of samples resolved the HWE violations and yielded similar effect estimates (Supplementary Table 7).

To place the right heart results into context with prior work, we compared right heart loci with those previously associated with left ventricular and aortic dimensions (Supplementary Figure 6) ${ }^{30,31}$. Among the right ventricular phenotypes, the RVESV was linked with the greatest number of loci (20). Of these, seven loci had previously been associated with the RVESV's left-heart counterpart (left ventricular end systolic volume; LVESV) at genome-wide significance. The BAG3 locus is the most strongly associated with both RVESV and LVESV. Both traits shared the same lead SNP: rs72840788, which has a near perfect correlation with a SNP, rs2234962, that leads to the missense change p.Cys151Arg in the BAG3 protein (Supplementary Figure $7)^{36}$.

In contrast, at the TTN locus, the RVESV lead SNP (rs955738, GWAS P = 4.3E-11) was in linkage equilibrium $\left(r^{2}=0.001\right)$ with the LVESV lead SNP ( $r$ 2562845, GWAS P = 1.3E-23). However, both SNPs were among the secondary signals for these traits: for LVESV, rs955738 was associated with $P=1.9 E-11$; for RVESV, rs2562845 was associated with $P=4.2 E-08$ (Supplementary Figure 8). It is possible that this distinction between primary association signals in the two ventricles is associated with differences in the regulation of TTN between the first (LV) and second (RV) heart fields, but establishing this will require additional investigation.

Among loci that were significant only for RVESV and not for LVESV, some, like the GATA4/CTSB locus, had a cluster of sub-threshold SNPs for LVESV. At this locus, the strongest LVESV-associated SNP ( $r$ 7012446, $P=1.5 E-06)$ was weakly correlated $\left(r^{2}=0.16\right)$ with the RVESV lead SNP (rs34015932, $\mathrm{P}=4$.1E-08), also suggesting allelic heterogeneity (Supplementary Figure 9). Other loci, such as that of OBSCN (encoding obscurin, a giant sarcomeric protein in the same family as titin), appeared to be right-ventricle specific, showing very little evidence of association with the left ventricle (Supplementary Figure 10).

\section{TWAS highlights role of WNT signaling in pulmonary root diameter}

Across all phenotypes, the strongest GWAS association was between the pulmonary root diameter and rs17608766 ( $\mathrm{P}=1.9 \mathrm{E}-51)$, near GOSR2. In a transcriptome-wide association study (TWAS) based on gene expression data from the aorta from GTEx v7 ${ }^{37}$, at the GOSR2 
locus we observed an association between pulmonary root diameter and WNT9B (full results in Supplementary Table 8). Interestingly, WNT9B is expressed in the endocardium overlying the heart valves during development, and loss of WNT9B leads to defective valve formation ${ }^{38}$. This locus was also recently shown to be linked with the mitral valve annular diameter ${ }^{39}$.

The strongest TWAS association for the proximal pulmonary artery diameter was with PDGFD, which is also the nearest gene to the lead SNP rs2128739. PDGFD loss-of-function variants were recently implicated in pulmonary hypertension in a sequencing-based case-control study ${ }^{40}$.

\section{Chamber-specific cell type enrichment}

To identify relevant cell types most relevant for the right atrial and right ventricular phenotypes, we performed stratified linkage disequilibrium (LD) score regression analysis that integrated single nucleus RNA-sequencing data from Tucker et $a{ }^{41}$. The strongest enrichment was seen between RVEF and right ventricular cardiomyocytes, while the strongest enrichment for the right atrial phenotypes was for vascular smooth muscle cell-like nuclei (Supplementary Figure 11).

\section{Rare variant association test}

Up to 13,523 individuals with imaging data had exome sequencing performed in the first batch of 50,000 exomes in the UK Biobank. After accounting for multiple testing, loss of function variants in one gene $(A A G A B)$ had significant association with the proximal pulmonary artery diameter (diameter larger by $0.42 \mathrm{~cm}$ on average among the 14 individuals with $A A G A B$ loss-offunction variants; $P=1.2 E-06$; Supplementary Figure 12). The AAGAB protein is involved in clathrin-mediated endocytosis ${ }^{42}$, and haploinsufficiency of $A A G A B$ has previously been reported to be associated with punctate palmoplantar keratoderma ${ }^{43,44}$. These prior reports are noteworthy in this context because of the association between palmoplantar keratoderma and $\mathrm{ARVC}^{45,46}$. Nevertheless, we did not identify an association between $A A G A B$ variants and RVEF $(\mathrm{P}=0.06)$ or RVESV $(\mathrm{P}=0.28)$. Additionally, common variants at the $A A G A B$ locus demonstrated no significant association with pulmonary artery diameter or right ventricular size and function in the GWAS. Future studies in additional populations will be required to assess the significance of the observed association between $A A G A B$ and right heart phenotypes.

\section{GWAS loci enriched in uncommon and difficult to phenotype cardiac}

\section{diseases}

To investigate the association between loci identified in this study and diseases that are not well represented (such as congenital heart diseases) or difficult to identify due to lack of specific diagnostic codes in the electronic health record (such as arrhythmogenic right ventricular cardiomyopathy), we performed proximity-based testing to assess enrichment of gene sets near the GWAS loci. We identified disease-related gene sets using the Open Targets platform (gene lists in Supplementary Table 9$)^{47}$ and asked whether more of those genes than expected by chance were found within $500 \mathrm{~kb}$ of the GWAS lead SNPs. Note that because the number of permutations generated by SNPSnap in the following tests was 10,000, the strongest possible association $P$ value was $1.0 \mathrm{E}-04^{48}$. 
The right atrial loci were in proximity to six Open Targets atrial septal defect-related genes (ACE, DMPK, MIR208A, NKX2-5, PLN, TBX5) with one-tailed permutation $\mathrm{P}=1.0 \mathrm{E}-04$. The right ventricular GWAS loci were in proximity to six ARVC-related genes ( $D S P, J U P$, $P P P 1 R 13 L, R B M 20, T M E M 43, T T N)$ with $\mathrm{P}=1.1 \mathrm{E}-03$. And the pulmonary artery loci were in proximity to 15 conotruncal abnormality-linked genes (BAZ1B, CEP152, DYNC2H1, ELN, EPHB4, FBN1, GATA4, KCNJ8, MECOM, NKX2-5, PDE3A, PDE5A, PLCE1, RYR1, $S M A R C A 4)$ with $\mathrm{P}=3.0 \mathrm{E}-04$ (Supplementary Figure 13).

We also analyzed a previously described panel of 129 cardiomyopathy-linked genes to contrast RVESV loci with LVESV loci ${ }^{30}$. Five of these genes were within a $500 \mathrm{~kb}$ radius of the RVESV loci; of these, three (BAG3, TMEM43, and TTN) had previously been found near genome-wide significant LVESV loci, while two (GATA4 and JUP) were not (Supplementary Figures 9 and 14). The RVESV and LVESV associations at the GATA4 locus have been described above. $J U P$, the gene that encodes plakoglobin, is a desmosomal protein that has also been associated with arrhythmogenic right ventricular cardiomyopathy and palmoplantar keratoderma, a syndrome known as Naxos disease ${ }^{11,13,49}$.

\section{Right heart polygenic scores are linked with cardiomyopathy and atrial fibrillation}

Finally, we assessed the association between polygenic scores derived from the right heart GWAS and incident cardiovascular diseases in UK Biobank participants unrelated to the individuals who underwent MRI.

A polygenic score for RVESV was associated with dilated cardiomyopathy (680 events and 409,944 non-events; HR 1.28 per SD; P = 2.4E-10; Figure 5). Notably, even after adjustment for the previously reported left ventricular end systolic volume BSA-indexed polygenic score ${ }^{30}$, the RVESV polygenic score remained associated with DCM (HR 1.17 per SD, P = 6.9E-05). Because of imprecision in clinical phenotyping from electronic health records (EHR), future work will be required to understand whether the RVESV polygenic score identifies additional cases of DCM that are driven by right ventricular dysfunction, or whether the score is identifying shared drivers of right- and left-ventricular dysfunction that were not ascertained in the left ventricular GWAS.

A polygenic score for the fractional area change of the right atrium in the four-chamber view was weakly inversely associated with the risk of atrial fibrillation or flutter (for 15,122 events and 402,951 non-events; HR 0.98 per SD; $P=1.9 E-03$ ). Results were similar when considering only atrial flutter as the outcome of interest (927 atrial flutter events and 423,824 non-events; HR 0.90 per SD; $P=1.2 \mathrm{E}-03$ ). We did not find a link between the risk of pulmonary hypertension (1,582 incident events and 423,136 non-events) and genetic predictions of proximal pulmonary artery diameter or pulmonary root diameter $(P=0.59$ and 0.93 , respectively). 


\section{Limitations}

This study is subject to several limitations. All data were derived from deep learning models of short axis or four-chamber long axis views from cardiovascular magnetic resonance imaging. These models have imprecision that would be reduced with further training data. Like any deep learning model, these models can fail and produce non physiologic measurements when presented with images that contain features not seen in the training data. An advantage of the semantic segmentation approach in this work is that outliers can be visually inspected and the model re-trained as needed. The right atrial measurements are two-dimensional estimates of a three-dimensional structure and therefore cannot capture complete information about atrial volume. The short axis images have a coarse $10 \mathrm{~mm}$ slice thickness which leads to partial volume imaging, which can be particularly difficult to visualize at the apex of the right ventricle, leading to under- or over-estimation by the deep learning model. Although we have attempted to correct for this by incorporating the higher resolution four-chamber long axis data during the surface reconstruction process, the correction itself can introduce additional artifacts: the image acquisitions for the short axis measurements are not simultaneous with one another or with the four-chamber long axis measurements, which can create misalignment (e.g., due to differences in breath holding) that introduces error when reconstructing the right ventricle. The deep learning models have not been tested outside of the specific devices and imaging protocols used by the UK Biobank and may not generalize to other data sets without additional finetuning. Participants' cardiac rhythm at the time of MRI (particularly normal sinus rhythm versus atrial fibrillation) was not adjudicated. The study population is largely of European ancestry, similar to the remainder of UK Biobank, limiting generalizability of the findings to other populations. The individuals who underwent MRI in the UK Biobank tend to be healthier than the remainder of the UK Biobank population, which itself is healthier than a general population. Finally, because we have used hospital-based ICD codes and procedural codes to identify individuals with disease, our study lacks an ARVC-specific analysis, and our disease definitions are susceptible to misclassification.

\section{Discussion}

We produced measurements of the right heart, including the right atrium, right ventricle, and pulmonary artery; analyzed their relationships with one another and with cardiovascular diseases; and identified 104 distinct genetic loci that are associated with these right-heart measurements. We drew several conclusions from these findings.

First, right heart phenotypes, including structural and functional measurements of the right atrium, right ventricle, and pulmonary artery, are heritable. While they share strong epidemiological and genetic correlation with the corresponding left heart structures, our findings of partial genetic correlation and distinct genome-wide significant loci also imply distinct drivers of variation between right and left heart structures. Developing a better understanding of these distinct drivers may ultimately permit more targeted therapies for right ventricle-predominant heart failure syndromes and primary cardiomyopathies such as ARVC. 
Second, we found that the GWAS loci were enriched for genes associated with developmental diseases such as atrial septal defect and conotruncal defects. In addition to the GWAS loci addressed above, several others were notable for connections to cardiovascular development. Right heart structures were associated with SNPs near NKX2-5, which plays a key role in maintaining the progenitor pool of cells of the secondary heart field ${ }^{5} ; T B X 3$, which controls the formation of the sinus node and loss of which leads to outflow tract malformations and septal defects $^{50,51}$; and $M Y L 4$, which encodes atrial light chain 1, missense variants in which have been linked to familial atrial fibrillation ${ }^{52}$.

Third, we observed links between right ventricular measurements-and polygenic predictions of these measurements - and disease. Individuals with pre-existing diagnoses of heart failure had reduced RVESV at the time for MRI, and those with pulmonary hypertension had markedly enlarged right ventricular volumes throughout the cardiac cycle (Figure 3). In the remainder of the population that was unrelated to those who underwent MRI, a polygenic predictor of RVESV was a strong predictor of a diagnosis of dilated cardiomyopathy (Figure 5). Notably, the RVESV polygenic score remained a significant predictor of dilated cardiomyopathy even after accounting for a previously reported genetic prediction of the left ventricle-implying a genetic basis for the role of right ventricular dysfunction in the pathogenesis of dilated cardiomyopathy. Consistent with emerging clinical evidence, this suggests that right ventricular structure and function are not merely of anthropomorphic interest, but actually represent endophenotypes for cardiomyopathy.

Fourth, despite our observation of strong epidemiological association between pulmonary hypertension and the proximal pulmonary diameter, we did not find an association between the polygenic predictor of proximal pulmonary diameter and the incidence of pulmonary hypertension. This lack of association may be because pulmonary hypertension in the UK Biobank is largely environmentally driven; may indicate that the genetic contributions to pulmonary artery diameter in disease-free individuals are not significantly associated with pulmonary artery pressure; or may be due to weak instrument bias.

Finally, machine learning enables the derivation of complex traits in a manner that is scalable. This permits biobank-scale investigation of previously understudied human phenotypes, such as measurements of the right atrium, right ventricle, and pulmonary artery; and promises to accelerate our understanding of cardiovascular disease.

\section{Online Methods}

\section{Study design}

Except where otherwise stated, all analyses were conducted in the UK Biobank, which is a richly phenotyped, prospective, population-based cohort that recruited 500,000 individuals aged 40-69 in the UK via mailer from 2006-2010 ${ }^{24}$. We analyzed 487,283 participants with genetic data who had not withdrawn consent as of February 2020. Access was provided under 
application \#7089 and approved by the Partners HealthCare institutional review board (protocol 2019P003144).

Here we provide an overview of the methods used in this manuscript that are explained in more detail below. We manually annotated pixels from magnetic resonance images from the UK Biobank: the pulmonary artery and the left and right ventricles were annotated in the short axis view, and the right atrium and right ventricle were annotated in the four-chamber long axis view. We then trained two deep learning models (one for each of the views) with our manual annotations, and applied this model to the remaining images in the UK Biobank. For the right ventricle, we integrated the data from the four chamber view and the short axis view to generate a surface mesh and derived the ventricular volumes from this mesh. We analyzed the relationships between each of these derived quantitative measurements of the right heart. We also analyzed their relationships with diseases and other phenotypes in the UK Biobank. Then, we excluded people with known disease and conducted genome-wide association studies of the right heart phenotypes. We performed transcriptome-wide association studies (TWAS) that incorporated publicly available gene expression data with our GWAS results to prioritize genes at most genomic loci. We analyzed the GWAS results in light of the four-chamber single nucleus sequencing data that is publicly available. We also performed a rare-variant association test in up to 13,523 UK Biobank participants with both imaging and exome sequencing data. Polygenic scores produced from SNPs associated with right heart phenotypes in the UK Biobank GWAS were used to predict incident atrial fibrillation or flutter, dilated cardiomyopathy, and pulmonary hypertension in the UK Biobank participants whose data did not contribute to the GWAS.

Statistical analyses were conducted with R version 3.6 (R Foundation for Statistical Computing, Vienna, Austria).

\section{Cardiovascular magnetic resonance imaging protocols}

At the time of this study, the UK Biobank had released images in over 45,000 participants of an imaging substudy that is ongoing ${ }^{22,23}$. Cardiovascular magnetic resonance imaging was performed with 1.5 Tesla scanners (Syngo MR D13 with MAGNETOM Aera scanners; Siemens Healthcare, Erlangen, Germany), and electrocardiographic gating for synchronization ${ }^{23}$. Several cardiac views were obtained. For this study, two views (the long axis four-chamber view and the short axis view) were used. In both of these views, balanced steady-state free precession cines, consisting of a series of 50 images throughout the cardiac cycle for each view, were acquired for each participant ${ }^{23}$. For the four-chamber images, only one imaging plane was available for each participant, with an imaging plane thickness of $6 \mathrm{~mm}$ and an average pixel width and height of $1.83 \mathrm{~mm}$. For the short axis view, several imaging planes were acquired. Starting at the base of the heart, $8 \mathrm{~mm}$-thick imaging planes were acquired with approximately $2 \mathrm{~mm}$ gaps between each plane, forming a stack perpendicular to the longitudinal axis of the left ventricle to capture the ventricular volume. For the short axis images, the average pixel width and height was $1.86 \mathrm{~mm}$. 


\section{Semantic segmentation and deep learning model training}

Semantic segmentation is the process of assigning labels to pixels of an image. Here, we labeled pixels within specific anatomical structures (the right atrial blood pool, the right ventricular blood pool, and the pulmonary artery blood pool), using a process similar to that described in our prior work evaluating the thoracic aorta ${ }^{31}$. Segmentation of cardiovascular structures was manually annotated in four-chamber and short axis images from the UK Biobank by a cardiologist (JP). To produce the model used in this manuscript, 714 short axis images were chosen, manually segmented, and used to train a deep learning model with PyTorch and fastai v1.0.61 ${ }^{25,53}$. The same was done separately with 445 four-chamber images. For both views, the models were based on a U-Net-derived architecture constructed with a ResNet34 encoder that was pre-trained on ImageNet ${ }^{26,54-56}$. The Adam optimizer was used ${ }^{57}$. The models were trained with a cyclic learning rate training policy ${ }^{58} .80 \%$ of the samples were used to train the model, and $20 \%$ were used for validation. Held-out test sets that were not used for training or validation were used to assess the final quality of both models.

Two separate models were trained: one for the short axis images, and one for the four-chamber long axis images. The hyperparameters used in their training are described below. For both models, random perturbations of the input images ("augmentations") were applied, including affine rotation, zooming, and modification of the brightness and contrast.

For the short axis images, all images were resized initially to $104 \times 104$ pixels during the first half of training, and then to $224 \times 224$ pixels during the second half of training. The model was trained with a mini-batch size of 16 (with small images) or 8 (with large images). Maximum weight decay was $1 \mathrm{E}-03$. The maximum learning rate was $1 \mathrm{E}-03$, chosen based on the learning rate finder ${ }^{25,59}$. Because the right ventricle and pulmonary artery blood pools occupied very little of the overall short axis image area, a focal loss function was used (with alpha 0.7 and gamma 0.7 ), which can improve performance in the case of imbalanced labels ${ }^{60}$. When training with small images, $60 \%$ of iterations were permitted to have an increasing learning rate during each epoch, and training was performed over 30 epochs while keeping the weights for all but the final layer frozen. Then, all layers were unfrozen, the learning rate was decreased to $1 \mathrm{E}-07$, and the model was trained for an additional 10 epochs. When training with large images, $30 \%$ of iterations were permitted to have an increasing learning rate, and training was done for 30 epochs while keeping all but the final layer frozen. Finally, all layers were unfrozen, the learning rate was decreased to $1 \mathrm{E}-07$, and the model was trained for an additional 10 epochs.

For the four-chamber long axis images, all images were resized initially to 76x104 pixels during the first half of training, and then to $150 \times 208$ pixels during the second half of training. The model was trained with a mini-batch size of 4 (with small images) or 2 (with large images). Maximum weight decay was $1 \mathrm{E}-02$. Cross entropy loss was used ${ }^{61} .30 \%$ of iterations were permitted to have an increasing learning rate during each epoch. When training with small images, the maximum learning rate was initially $1 \mathrm{E}-03$, and training was performed over 50 epochs while keeping all weights frozen except for the final layer. Then, all layers were unfrozen, the learning rate was decreased to $3 \mathrm{E}-05$, and the model was trained for an additional 15 epochs. When training with large images, the maximum learning rate was set to $3 \mathrm{E}-04$, and the model was 
trained for 50 epochs while keeping all but the final layer frozen. Finally, all layers were unfrozen, the learning rate was decreased to $1 \mathrm{E}-07$, and the model was retrained for an additional 15 epochs.

The final short axis and four-chamber long axis models were then applied, respectively, to all available short axis images and four-chamber long axis images available in the UK Biobank as of November 2020.

\section{Deep learning model output quality control}

Accuracy of the two deep learning models was assessed with additional manually annotated images that were not used for model training or validation, with each annotation category (such as pulmonary artery blood pool) evaluated based on the Sørensen-Dice coefficient ${ }^{62,63}$, which scales from 0 (no agreement between manual and automated annotations) to 1 (perfect agreement). Images with no pixels assigned to a feature by either the truth labels or the deep learning model output were assigned to have a Dice coefficient of 1.

\section{Right atrial measurements from the four-chamber long axis view}

Three long-axis views were obtained in the UK Biobank (two-chamber, three-chamber, and fourchamber). Of these, only the four-chamber view reliably captures the right atrium. We therefore treated the right atrium as a planar surface, counting the pixels that were labeled by the four chamber semantic segmentation model as right atrium, and multiplying that number by the height and width of each pixel to obtain a right atrial area (with units of $\mathrm{cm}^{2}$ ). For each individual, we obtained the maximum atrial area, the minimum atrial area, and the fractional area change (maximum area minus minimum area, divided by maximum area).

\section{Pulmonary artery measurements from the short axis view}

For most individuals in the UK Biobank, the pulmonary artery can be readily visualized in the basal-most short axis imaging planes. When the pulmonary artery was visible in multiple imaging planes, we measured the artery in both the basal-most and the apical-most plane that still captured a pulmonary artery cross-section. To facilitate reproducibility, we only evaluated images from the frame representing ventricular end-systole. We refer to the basal-most segment of pulmonary artery as the "proximal pulmonary artery" and the apical-most segment that sits just basal to the right ventricular outflow tract as the "pulmonary root."

The proximal pulmonary artery and pulmonary root were treated as ellipses. We computed major and minor axes using classical image moment algorithms ${ }^{31,64}$. For both the proximal pulmonary artery and the pulmonary root, the length of the minor elliptical axis (i.e., the diameter) was computed. We excluded any measurements where the artery was divided into more than one connected component ${ }^{65}$. For the proximal pulmonary artery, we permitted elliptical eccentricity values of up to 0.86 (where eccentricity is $\sqrt{1-\frac{b^{2}}{a^{2}}}$, with a being the elliptical major axis and $b$ being the elliptical minor axis). We permitted a liberal eccentricity cutoff 
because a common cause of high eccentricity in the proximal pulmonary artery images was outof-plane curvature of the proximal pulmonary artery, which erroneously elongates the major elliptical axis but does not significantly affect the minor elliptical axis (which we are using as the diameter). For the pulmonary artery root, we required an eccentricity below 0.77 . We used a more stringent cutoff at the root because a common cause of high eccentricity in these images was partial imaging of the right ventricle, which can erroneously foreshorten the minor elliptical axis. In addition, we excluded images where the cross-sectional area of the pulmonary artery was less than $2 \mathrm{~cm}^{2}$.

\section{Right ventricular annotation and surface reconstruction integrating long- and short-axis data}

The right ventricle was visible in multiple views, permitting a reconstruction of its volume through the incorporation of data from multiple images. Stacked together, images from the short axis view provided a complete 3-dimensional representation of the right ventricle; however, this stack had a coarse $10 \mathrm{~mm}$ resolution along the length of the right ventricle from base to apex. In contrast, the four-chamber long axis view had approximately $2 \mathrm{~mm}$ resolution along the same axis. To take advantage of the strengths of both sources of data, we reconstructed the surface of the right ventricle using a Poisson surface reconstruction technique described in detail below. This enabled the computation of right ventricular end diastolic volume, end systolic volume, stroke volume, and ejection fraction.

To produce consistent estimates of the RV volumes throughout the cardiac cycle, we integrated information from the long- and short-axis segmentations by reconstructing 3-dimensional surfaces enclosing the RV cavity. We first used image metadata from the standard Image Position (Patient) [0020,0032] and Image Orientation (Patient) [0020,0037] DICOM tags to corotate the 4-chamber and short-axis slices into the same reference system. Then, we implemented a custom reconstruction routine based on the Poisson algorithm to generate surfaces that fitted through the boundaries of the RV segmentations ${ }^{66}$. As the Poisson algorithm requires local curvature as an input, we specified for the surface normal directions to lie onto the plane of the MRI slices and to be locally oriented towards either the pericardium (at the free wall) or the left ventricle (at the interventricular septum). The reconstructed RVs were then postprocessed to correct for eventual artifacts in the basal short-axis slices, where the segmentation model may occasionally mistake the right atrium for part of the RV. Leveraging the fine resolution of the long-axis CMR in the apex-to-base direction, we discarded the portions of the reconstructed $\mathrm{RV}$ s that overextended above the plane separating the long-axis segmentations of the right atrium and of the RV (i.e., approximately co-aligned with the tricuspid valve plane). Finally, the RV volumes were estimated from the reconstructed surfaces using a discrete version of the divergence theorem, as implemented in the open-source VTK library (Kitware Inc.).

\section{Phenotypic characterization of right heart structure and function}

Using $R$ version 3.6, we evaluated the mean and standard deviation of the right heart measurements, described them in age- and sex-stratified tables, and created sex-stratified 
kernel density plots with ggplot ${ }^{67}$. We computed the Pearson correlation between all right heart phenotypes and available left heart phenotypes that were previously described ${ }^{30,31}$.

We tested for association between the right heart phenotypes and PheCode-based disease labels derived from ICD-10 codes and OPCS-4 codes that were present prior to each participant's magnetic resonance imaging date ${ }^{32}$. Association between each disease code and the right heart phenotypes was performed with linear models accounting for the MRI serial number, sex, the first five principal components of ancestry, age at enrollment, the cubic natural spline of age at the time of MRI, and the genotyping array. Splines were not placed on age at enrollment because of its collinearity with age at the time of MRI.

We used three custom disease definitions to focus on chamber-specific disease relationships (atrial fibrillation with RA FAC; heart failure with RVESV; and pulmonary hypertension with the proximal pulmonary artery diameter; defined in Supplementary Table 3). Association between each disease (as a binary independent variable) and the right heart phenotypes (as the dependent variable) was performed using a linear model that also accounted for the MRI serial number, sex, the first five principal components of ancestry, age at enrollment, the cubic natural spline of age at the time of MRI, and the genotyping array. As above, splines were not placed on age at enrollment because of its collinearity with age at the time of MRI.

We also modeled the association between three diseases (pulmonary hypertension, heart failure, and cataract) and right ventricular volume throughout the cardiac cycle. The magnetic resonance images were acquired as a series of 50 images throughout a cardiac cycle, and so our Poisson surface reconstruction yielded right ventricular volume for each of these timepoints (one-fiftieth of a cardiac cycle). At each of these timepoints, we used a linear model to test the association between the right ventricular volume (independent variable) and the presence or absence of each of the three diseases, as well as covariates that included the heart rate at the time of MRI, weight, height, age at enrollment, the cubic natural spline of age at the time of MRI, sex, genotyping array, and the first five principal components of ancestry. In the results, we report the $P$ value for the linear model regression coefficient for the disease. To model the estimated volume for individuals with or without disease, we compute the output of the linear model for a 55-year-old woman who enrolled 5 years previously in the UK Biobank, 162 centimeters tall and weighing 75.6 kilograms. We then toggle the presence or absence of disease in the model to obtain volumes with or without disease, fixing other covariates.

\section{Genotyping, imputation, and genetic quality control}

UK Biobank samples were genotyped on either the UK BiLEVE or UK Biobank Axiom arrays and imputed into the Haplotype Reference Consortium panel and the UK10K+1000 Genomes panel $^{68}$. Variant positions were keyed to the GRCh37 human genome reference. Genotyped variants with genotyping call rate $<0.95$ and imputed variants with INFO score $<0.3$ or minor allele frequency $<=0.005$ in the analyzed samples were excluded. After variant-level quality control, 11,631,796 imputed variants remained for analysis. 
Participants without imputed genetic data, or with a genotyping call rate $<0.98$, mismatch between self-reported sex and sex chromosome count, sex chromosome aneuploidy, excessive third-degree relatives, or outliers for heterozygosity were excluded from genetic analysis ${ }^{68}$. Participants were also excluded from genetic analysis if they had a history of pulmonary hypertension, atrial fibrillation, heart failure, or coronary artery disease documented by ICD code or procedural code from the inpatient setting prior to the time they underwent cardiovascular magnetic resonance imaging at a UK Biobank assessment center. Our definitions of these diseases in the UK Biobank are provided in Supplementary Table 3.

\section{Heritability and genome-wide association analyses}

We analyzed nine primary right heart phenotypes. For the right atrium, we assessed maximum area, minimum area, and fractional area change. For the right ventricle, we assessed end diastolic volume, end systolic volume, stroke volume, and ejection fraction. For the pulmonary system, we assessed the diameter of the pulmonary root and the proximal pulmonary artery. In addition, we analyzed body surface area-indexed values for all areas and volumes (i.e., excluding RA FAC and RVEF which are dimensionless). In total, we conducted 16 genome-wide association studies with these traits.

BOLT-REML v2.3.4 was used to assess the SNP-heritability of the phenotypes, as well as their genetic correlation with one another using the directly genotyped variants in the UK Biobank ${ }^{33}$.

Before conducting genome-wide association studies, a rank-based inverse normal transformation was applied to the quantitative right heart traits ${ }^{69}$. All traits were adjusted for age at enrollment, age and $\mathrm{age}^{2}$ at the time of MRI, the first 10 principal components of ancestry, sex, the genotyping array, and the MRI scanner's unique identifier.

Genome-wide association studies for each phenotype were conducted using BOLT-LMM version 2.3.4 to account for cryptic population structure and sample relatedness ${ }^{33,34}$. We used the full autosomal panel of 714,558 directly genotyped SNPs that passed quality control to construct the genetic relationship matrix (GRM), with covariate adjustment as noted above. Associations on the $X$ chromosome were also analyzed, using all autosomal SNPs and $X$ chromosomal SNPs to construct the GRM (N=732,193 SNPs), with the same covariate adjustments and significance threshold as in the autosomal analysis. In this analysis mode, BOLT treats individuals with one $X$ chromosome as having an allelic dosage of $0 / 2$ and those with two $X$ chromosomes as having an allelic dosage of $0 / 1 / 2$. Variants with association $P<$ $5 \cdot 10^{-8}$, a commonly used threshold, were considered to be genome-wide significant. In addition, we used a secondary threshold of $P<3.1 \cdot 10^{-9}\left(5 \cdot 10^{-8}\right.$ divided by 16 phenotypes $)$ to identify associations that were study-wide significant.

We identified lead SNPs for each trait. Linkage disequilibrium (LD) clumping was performed with PLINK-1.970 using the same participants used for the GWAS, rather than a generic reference panel. We outlined a 5-megabase window (--clump-kb 5000) and used a stringent LD threshold (--r2 0.001) in order to account for long LD blocks such as those near the Williams-Beuren locus on chromosome 7 and the Noonan syndrome locus on chromosome $12^{71-73}$. With the 
independently significant clumped SNPs, distinct genomic loci were then defined by starting with the SNP with the strongest $P$ value, excluding other SNPs within $500 \mathrm{~kb}$, and iterating until no SNPs remained. Independently significant SNPs that defined each genomic locus are termed the lead SNPs.

Lead SNPs were tested for deviation from Hardy-Weinberg equilibrium (HWE) at a threshold of $P<1 E-06^{70}$. To assess whether the HWE violations affected the association signals, SNPs with HWE P < 1E-06 were re-analyzed with R's glm after excluding samples that were not within the UK Biobank's centrally-adjudicated "white British" subset, using the same covariates as the BOLT-LMM model.

Linkage disequilibrium (LD) score regression analysis was performed using Idsc version 1.0.0 ${ }^{35}$. With Idsc, the genomic control factor (lambda GC) was partitioned into components reflecting polygenicity and inflation, using the software's defaults.

\section{Transcriptome-wide association study}

For each phenotype, we performed a TWAS to identify correlated genes based on imputed cisregulated gene expression ${ }^{74-76}$. We used FUSION with eQTL data from GTEx v7. Precomputed transcript expression reference weights for the aorta (used for the pulmonary artery traits), left ventricle (used for the right ventricular traits), and right atrial appendage (used for the right atrial traits) were obtained from the FUSION authors' website (http://gusevlab.org/projects/fusion/) $)^{37,75}$. FUSION was then run with its default settings.

\section{Stratified LD Score Regression}

To identify putative cell types most relevant for each GWAS trait, we performed stratified linkage disequilibrium (LD) score regression analysis using single nucleus RNA-sequencing data from Tucker et $a /^{35,41,77}$. Cell type specific markers within the RA and RV were calculated separately for the 9 main cell types using a limma-voom differential expression model on aggregated counts per individual ${ }^{78}$. Only individuals with greater than 25 nuclei of a given cell type were considered. Genes were sorted by t statistic per cell type and the top $90 \%$ of genes were used to generate LD Score Regression annotations ${ }^{77}$. SNPs within $100 \mathrm{~KB}$ of any gene from a specific cell type were annotated for the respective cell type using 1000 Genomes European individuals ${ }^{79}$. We then performed stratified LD score regression with these annotations in combination with the baseline model described in Finucane, et al, 2015, only including high quality HapMap3 SNPs ${ }^{80}$. We used the RA cell type specific annotations and RV cell type specific annotations for the RA and RV specific GWAS traits, respectively.

\section{Exome sequencing}

We conducted an exome sequencing analysis in the first 50,000 exomes released by the UK Biobank. Exome sequencing was performed by Regeneron and reprocessed centrally by the UK Biobank following the Functional Equivalent pipeline ${ }^{81}$. Exomes were captured with the IDT xGen Exome Research Panel v1.0, and sequencing was performed with 75-base paired-end 
reads on the Illumina NovaSeq 6000 platform using S2 flowcells. Alignment to GRCh38 was performed centrally with BWA-mem ${ }^{82}$. Variant calling was performed centrally with GATK $3.0^{83}$. Variants were hard-filtered if the inbreeding coefficient was $<-0.03$, or if none of the following were true: read depth was greater than or equal to 10; genotype quality was greater than or equal to 20; or allele balance was greater than or equal to 0.2 . Variants were annotated with the Ensembl Variant Effect Predictor version 95 using the --pick-allele flag ${ }^{84}$. LOFTEE 1.0 was used to identify high-confidence loss of function variants: stop-gain, splice-site disrupting, and frameshift variants ${ }^{85}$. In total, 49,997 exomes were available, of which a subset overlapped with the participants who had undergone magnetic resonance imaging: 12,420 with right atrial measurements, 12,699 with right ventricular measurements, and 13,523 pulmonary artery measurements.

\section{Rare variant association test}

We conducted a collapsing burden test to assess the impact of loss-of-function variants in up to 13,523 participants who had undergone exome sequencing data and magnetic resonance imaging. Variants with MAF $>=0.001$ were excluded. We excluded genes with fewer than 10 loss-of-function variants passing the above criteria. The models testing for association between loss-of-function in each gene and the right heart traits were adjusted for weight $(\mathrm{kg})$, height $(\mathrm{cm})$, body mass index $\left(\mathrm{kg} / \mathrm{m}^{2}\right)$, the MRI serial number, age at enrollment, the cubic natural spline of age at the time of MRI, sex, the genotyping array, and the first five principal components of ancestry. As above, splines were not placed on age at enrollment because of its collinearity with age at the time of MRI.

\section{Open Targets gene set enrichment at GWAS loci}

Using the Open Targets platform, we created gene sets corresponding to ARVC, atrial fibrillation, atrial septal defect, and transposition of the great arteries (TGA) or conotruncal anomaly by fetching all genes with an overall association score of 0.05 or greater (Supplementary Table 9) ${ }^{47}$. Using SNPsnap, we generated 10,000 sets of SNPs that matched the lead SNPs based on parameters including minor allele frequency, SNPs in linkage disequilibrium, distance from the nearest gene, and gene density. We counted the number of OpenTargets genes within $500 \mathrm{~kb}$ of the lead SNPs from our study. We then repeated the same procedure for each of the 10,000 synthetic SNPsnap lead SNP lists, to set a neutral expectation for the number of overlapping genes based on chance. This allowed us to compute one-tailed permutation $\mathrm{P}$ values for each group of disease genes (with the most extreme possible $\mathrm{P}$ value based on 10,000 randomly chosen sets of SNPs being $\left.1 \cdot 10^{-4}\right)$.

\section{Polygenic risk analysis}

We computed a polygenic score based on 21 clumped, genome-wide significant SNPs (of which 20 were lead SNPs) from the RVESV GWAS. We applied this score to the entire UK Biobank population, after excluding any participant who had undergone imaging or who was related within 3 degrees to individuals with imaging. We analyzed the relationship between this polygenic prediction of the RVESV and dilated cardiomyopathy using a Cox proportional 
hazards model as implemented by the R survival package ${ }^{86}$. We excluded individuals with disease that was diagnosed prior to enrollment in the UK Biobank. We counted survival as the number of years between enrollment and disease diagnosis (for those with disease) and those being censored due to death, loss to follow-up, or end of follow-up time. We adjusted for covariates including sex, the cubic basis spline of age at enrollment, the interaction between the cubic basis spline of age at enrollment and sex, the genotyping array, the first five principal components of ancestry, and the cubic basis splines of height $(\mathrm{cm})$, weight $(\mathrm{kg}), \mathrm{BMI}\left(\mathrm{kg} / \mathrm{m}^{2}\right)$, diastolic blood pressure, systolic blood pressure.

The same procedure was performed to produce a 5-SNP polygenic score for the RA fractional area change (all of which were lead SNPs) that was tested for association with atrial fibrillation and flutter. And the procedure was repeated to produce a 42-SNP polygenic score for the pulmonary artery diameter (of which 38 were lead SNPs) that was tested for association with pulmonary hypertension. The SNP weights are available in Supplementary Table 10.

To assess the impact of the RVESV PRS even after accounting for the previously reported LVESVi PRS, we also added the LVESVi PRS as a covariate to the RVESV-dilated cardiomyopathy Cox model.

\section{Data availability}

UK Biobank data are made available to researchers from research institutions with genuine research inquiries, following IRB and UK Biobank approval. GWAS summary statistics will be available upon publication at the Broad Institute Cardiovascular Disease Knowledge Portal ( http://www.broadcvdi.org ). All other data are contained within the article and its supplementary information, or are available upon reasonable request to the corresponding author.

\section{Code availability}

The code used to perform Poisson surface reconstruction from segmentation output is located at https://github.com/broadinstitute/ml4h and is available under an open-source BSD license. The code used to perform permutation testing to assess enrichment of disease-related genes near GWAS loci is located at https://github.com/carbocation/genomisc and is available under an open-source BSD license.

\section{Author contributions}

JPP and PTE conceived of the study. JPP and VN annotated images. JPP trained the deep learning models. PD performed surface reconstruction. JPP, VN, MDC, PD, SNF, and MDRK conducted bioinformatic analyses. JPP and PTE wrote the paper. All other authors contributed to the analysis plan or provided critical revisions. 


\section{Sources of funding}

This work was supported by the Fondation Leducq (14CVD01), and by grants from the National Institutes of Health to Dr. Ellinor (1RO1HL092577, R01HL128914, K24HL105780) and Dr. Ho (R01HL134893, R01HL140224, K24HL153669). This work was supported by a John S LaDue Memorial Fellowship and the Sarnoff Cardiovascular Research Foundation Scholar Award to Dr. Pirruccello. Dr. Nauffal is supported by NIH grant 5T32HL007604-35. Dr. Khurshid is supported by NIH grant T32HL007208. Dr. Lubitz is supported by NIH grant 1R01HL139731 and American Heart Association 18SFRN34250007. This work was supported by a grant from the American Heart Association Strategically Focused Research Networks to Dr. Ellinor. Dr. Lindsay is supported by the Fredman Fellowship for Aortic Disease and the Toomey Fund for Aortic Dissection Research. This work was funded by a collaboration between the Broad Institute and IBM Research.

\section{Disclosures}

Drs. Pirruccello has served as a consultant for Maze Therapeutics. Dr. Batra is supported by grants from Bayer AG and IBM applying machine learning in cardiovascular disease. Dr. Lubitz receives sponsored research support from Bristol Myers Squibb / Pfizer, Bayer AG, Boehringer Ingelheim, and Fitbit, and has consulted for Bristol Myers Squibb / Pfizer and Bayer AG, and participates in a research collaboration with IBM. Dr. Ng is employed by IBM Research. Dr. Ho is supported by a grant from Bayer AG focused on machine-learning and cardiovascular disease and a research grant from Gilead Sciences. Dr. Ho has received research supplies from EcoNugenics. Dr. Philippakis is employed as a Venture Partner at GV; he is also supported by a grant from Bayer AG to the Broad Institute focused on machine learning for clinical trial design. Dr. Ellinor is supported by a grant from Bayer AG to the Broad Institute focused on the genetics and therapeutics of cardiovascular diseases. Dr. Ellinor has also served on advisory boards or consulted for Bayer AG, Quest Diagnostics, MyoKardia and Novartis. Remaining authors report no disclosures.

\section{References}

1. Olson, E. N. Gene Regulatory Networks in the Evolution and Development of the Heart.

Science 313, 1922-1927 (2006).

2. Koshiba-Takeuchi, K. et al. Reptilian heart development and the molecular basis of cardiac chamber evolution. Nature 461, 95-98 (2009).

3. Farmer, C. G. Evolution of the Vertebrate Cardio-Pulmonary System. Annual Review of Physiology 61, 573-592 (1999).

4. Galli, D. et al. Atrial myocardium derives from the posterior region of the second heart field, 
which acquires left-right identity as Pitx2c is expressed. Development 135, 1157-1167 (2008).

5. Meilhac, S. M. \& Buckingham, M. E. The deployment of cell lineages that form the mammalian heart. Nature Reviews Cardiology 15, 705-724 (2018).

6. Verzi, M. P., McCulley, D. J., De Val, S., Dodou, E. \& Black, B. L. The right ventricle, outflow tract, and ventricular septum comprise a restricted expression domain within the secondary/anterior heart field. Dev Biol 287, 134-145 (2005).

7. Zaffran, S., Kelly, R. G., Meilhac, S. M., Buckingham, M. E. \& Brown, N. A. Right ventricular myocardium derives from the anterior heart field. Circ Res 95, 261-268 (2004).

8. Jiang, X., Rowitch, D. H., Soriano, P., McMahon, A. P. \& Sucov, H. M. Fate of the mammalian cardiac neural crest. Development 127, 1607-1616 (2000).

9. Li, J., Chen, F. \& Epstein, J. A. Neural crest expression of Cre recombinase directed by the proximal Pax3 promoter in transgenic mice. genesis 26, 162-164 (2000).

10. Lin, C.-J., Lin, C.-Y., Chen, C.-H., Zhou, B. \& Chang, C.-P. Partitioning the heart: mechanisms of cardiac septation and valve development. Development 139, 3277-3299 (2012).

11. McKoy, G. et al. Identification of a deletion in plakoglobin in arrhythmogenic right ventricular cardiomyopathy with palmoplantar keratoderma and woolly hair (Naxos disease). The Lancet 355, 2119-2124 (2000).

12. McNally, E., MacLeod, H. \& Dellefave-Castillo, L. Arrhythmogenic Right Ventricular Cardiomyopathy. in GeneReviews ${ }^{\circledR}$ (eds. Adam, M. P. et al.) (University of Washington, Seattle, 1993).

13. Protonotarios, N. \& Tsatsopoulou, A. Naxos disease: Cardiocutaneous syndrome due to cell adhesion defect. Orphanet Journal of Rare Diseases 1, 4 (2006).

14. Romero, J., Mejia-Lopez, E., Manrique, C. \& Lucariello, R. Arrhythmogenic Right Ventricular Cardiomyopathy (ARVC/D): A Systematic Literature Review. Clin Med Insights Cardiol 7, 
CMC.S10940 (2013).

15. Ghio, S. et al. Independent and additive prognostic value of right ventricular systolic function and pulmonary artery pressure in patients with chronic heart failure. J Am Coll Cardiol 37, 183-188 (2001).

16. Kjaergaard, J. et al. Right ventricular dysfunction as an independent predictor of short- and long-term mortality in patients with heart failure. Eur J Heart Fail 9, 610-616 (2007).

17. Melenovsky, V., Hwang, S.-J., Lin, G., Redfield, M. M. \& Borlaug, B. A. Right heart dysfunction in heart failure with preserved ejection fraction. Eur Heart $\mathrm{J}$ 35, 3452-3462 (2014).

18. Borlaug, B. A., Kane, G. C., Melenovsky, V. \& Olson, T. P. Abnormal right ventricularpulmonary artery coupling with exercise in heart failure with preserved ejection fraction. Eur Heart J 37, 3293-3302 (2016).

19. Gorter, T. M. et al. Right heart dysfunction and failure in heart failure with preserved ejection fraction: mechanisms and management. Position statement on behalf of the Heart Failure Association of the European Society of Cardiology. Eur J Heart Fail 20, 16-37 (2018).

20. Ho, J. E. et al. Exercise Pulmonary Hypertension Predicts Clinical Outcomes in Patients With Dyspnea on Effort. J Am Coll Cardiol 75, 17-26 (2020).

21. Mishra, S. \& Kass, D. A. Cellular and molecular pathobiology of heart failure with preserved ejection fraction. Nature Reviews Cardiology 1-24 (2021) doi:10.1038/s41569-020-00480-6.

22. Petersen, S. E. et al. Imaging in population science: cardiovascular magnetic resonance in 100,000 participants of UK Biobank - rationale, challenges and approaches. Journal of Cardiovascular Magnetic Resonance 15, 46 (2013).

23. Petersen, S. E. et al. UK Biobank's cardiovascular magnetic resonance protocol. J Cardiovasc Magn Reson 18, (2016).

24. Sudlow, C. et al. UK biobank: an open access resource for identifying the causes of a wide range of complex diseases of middle and old age. PLoS medicine 12, e1001779 (2015). 
25. Howard, J. \& Gugger, S. Fastai: A Layered API for Deep Learning. Information 11, 108 (2020).

26. Ronneberger, O., Fischer, P. \& Brox, T. U-Net: Convolutional Networks for Biomedical Image Segmentation. arXiv:1505.04597 [cs] (2015).

27. Maceira, A. M., Cosín-Sales, J., Roughton, M., Prasad, S. K. \& Pennell, D. J. Reference right atrial dimensions and volume estimation by steady state free precession cardiovascular magnetic resonance. J Cardiovasc Magn Reson 15, 29 (2013).

28. Burman, E. D., Keegan, J. \& Kilner, P. J. Pulmonary artery diameters, cross sectional areas and area changes measured by cine cardiovascular magnetic resonance in healthy volunteers. Journal of Cardiovascular Magnetic Resonance 18, 12 (2016).

29. Foppa, M. et al. Right Ventricular Volumes and Systolic Function by Cardiac Magnetic Resonance and the Impact of Sex, Age, and Obesity in a Longitudinally Followed Cohort Free of Pulmonary and Cardiovascular Disease: The Framingham Heart Study. Circ Cardiovasc Imaging 9, e003810 (2016).

30. Pirruccello, J. P. et al. Analysis of cardiac magnetic resonance imaging in 36,000 individuals yields genetic insights into dilated cardiomyopathy. Nature Communications 11, 1-10 (2020).

31. Pirruccello, J. P. et al. Deep learning enables genetic analysis of the human thoracic aorta. bioRxiv 2020.05.12.091934 (2020) doi:10.1101/2020.05.12.091934.

32. Wu, P. et al. Mapping ICD-10 and ICD-10-CM Codes to Phecodes: Workflow Development and Initial Evaluation. JMIR Med Inform 7, e14325 (2019).

33. Loh, P.-R. et al. Efficient Bayesian mixed-model analysis increases association power in large cohorts. Nature Genetics 47, 284-290 (2015).

34. Loh, P.-R., Kichaev, G., Gazal, S., Schoech, A. P. \& Price, A. L. Mixed-model association for biobank-scale datasets. Nature Genetics 50, 906-908 (2018).

35. Bulik-Sullivan, B. K. et al. LD Score regression distinguishes confounding from polygenicity 
in genome-wide association studies. Nature Genetics 47, 291-295 (2015).

36. Boughton, A. P. et al. LocusZoom.js: Interactive and embeddable visualization of genetic association study results. bioRxiv 2021.01.01.423803 (2021) doi:10.1101/2021.01.01.423803.

37. Lonsdale, J. et al. The Genotype-Tissue Expression (GTEx) project. Nature Genetics 45, 580-585 (2013).

38. Goddard, L. M. et al. Hemodynamic forces sculpt developing heart valves through a KLF2WNT9B paracrine signaling axis. Dev Cell 43, 274-289.e5 (2017).

39. Yu, M. et al. Computational estimates of mitral annular diameter in systole and diastole cardiac cycle reveal novel genetic determinants of valve function and disease. medRxiv 2020.12.02.20242206 (2020) doi:10.1101/2020.12.02.20242206.

40. Zhu, N. et al. Rare variant analysis of 4,241 pulmonary arterial hypertension cases from an international consortium implicate FBLN2, PDGFD and rare de novo variants in PAH. bioRxiv 2020.05.29.124255 (2020) doi:10.1101/2020.05.29.124255.

41. Tucker Nathan R. et al. Transcriptional and Cellular Diversity of the Human Heart. Circulation 142, 466-482 (2020).

42. Gulbranson, D. R. et al. AAGAB Controls AP2 Adaptor Assembly in Clathrin-Mediated Endocytosis. Developmental Cell 50, 436-446.e5 (2019).

43. Giehl, K. A. et al. Nonsense mutations in AAGAB cause punctate palmoplantar keratoderma type Buschke-Fischer-Brauer. Am J Hum Genet 91, 754-759 (2012).

44. Pohler, E. et al. Haploinsufficiency for AAGAB causes clinically heterogeneous forms of punctate palmoplantar keratoderma. Nature Genetics 44, 1272-1276 (2012).

45. Simpson, M. A. et al. Homozygous mutation of desmocollin-2 in arrhythmogenic right ventricular cardiomyopathy with mild palmoplantar keratoderma and woolly hair. Cardiology 113, 28-34 (2009).

46. Yao, J. V. \& Winship, I. More than meets the eye: Palmoplantar keratoderma and 
arrhythmogenic right ventricular cardiomyopathy in a patient with loss of the DSP gene. JAAD Case Rep 6, 804-806 (2020).

47. Mountjoy, E. et al. Open Targets Genetics: An open approach to systematically prioritize causal variants and genes at all published GWAS trait-associated loci. bioRxiv 2020.09.16.299271 (2020) doi:10.1101/2020.09.16.299271.

48. Pers, T. H., Timshel, P. \& Hirschhorn, J. N. SNPsnap: a Web-based tool for identification and annotation of matched SNPs. Bioinformatics 31, 418-420 (2015).

49. Protonotarios, N. et al. Cardiac abnormalities in familial palmoplantar keratosis. Br Heart J 56, 321-326 (1986).

50. Bakker Martijn L. et al. Transcription Factor Tbx3 Is Required for the Specification of the Atrioventricular Conduction System. Circulation Research 102, 1340-1349 (2008).

51. Hoogaars, W. M. H. et al. Tbx3 controls the sinoatrial node gene program and imposes pacemaker function on the atria. Genes Dev 21, 1098-1112 (2007).

52. Orr, N. et al. A mutation in the atrial-specific myosin light chain gene (MYL4) causes familial atrial fibrillation. Nat Commun 7, 11303 (2016).

53. Paszke, A. et al. PyTorch: An Imperative Style, High-Performance Deep Learning Library. arXiv:1912.01703 [cs, stat] (2019).

54. Deng, J. et al. ImageNet: A large-scale hierarchical image database. in 2009 IEEE Conference on Computer Vision and Pattern Recognition 248-255 (2009). doi:10.1109/CVPR.2009.5206848.

55. He, K., Zhang, X., Ren, S. \& Sun, J. Deep Residual Learning for Image Recognition. arXiv:1512.03385 [cs] (2015).

56. Krizhevsky, A., Sutskever, I. \& Hinton, G. E. ImageNet Classification with Deep Convolutional Neural Networks.

57. Kingma, D. P. \& Ba, J. Adam: A Method for Stochastic Optimization. arXiv:1412.6980 [cs] (2017). 
58. Smith, L. N. Cyclical Learning Rates for Training Neural Networks. arXiv:1506.01186 [cs] (2015).

59. Smith, L. N. A disciplined approach to neural network hyper-parameters: Part 1 -- learning rate, batch size, momentum, and weight decay. arXiv:1803.09820 [cs, stat] (2018).

60. Lin, T.-Y., Goyal, P., Girshick, R., He, K. \& Dollár, P. Focal Loss for Dense Object Detection. arXiv:1708.02002 [cs] (2018).

61. Cox, D. R. The Regression Analysis of Binary Sequences. Journal of the Royal Statistical Society: Series B (Methodological) 20, 215-232 (1958).

62. Dice, L. R. Measures of the Amount of Ecologic Association Between Species. Ecology 26, 297-302 (1945).

63. Sørensen, T. J. A method of establishing groups of equal amplitude in plant sociology based on similarity of species content and its application to analyses of the vegetation on Danish commons. (I kommission hos E. Munksgaard, 1948).

64. Horn, B. Robot vision. (MIT Press ; McGraw-Hill, 1986).

65. Rosenfeld, A. \& Pfaltz, J. L. Sequential Operations in Digital Picture Processing. J. ACM 13, 471-494 (1966).

66. Kazhdan, M. \& Hoppe, H. Screened poisson surface reconstruction. ACM Trans. Graph. 32, 29:1-29:13 (2013).

67. Wickham, H. ggplot2: Elegant Graphics for Data Analysis. (Springer-Verlag, 2009). doi:10.1007/978-0-387-98141-3.

68. Bycroft, C. et al. The UK Biobank resource with deep phenotyping and genomic data. Nature 562, 203 (2018).

69. Yang, J. et al. FTO genotype is associated with phenotypic variability of body mass index. Nature 490, 267-272 (2012).

70. Chang, C. C. et al. Second-generation PLINK: rising to the challenge of larger and richer datasets. Gigascience 4, (2015). 
71. Osborne, L. R. \& Mervis, C. B. Rearrangements of the Williams-Beuren syndrome locus: molecular basis and implications for speech and language development. Expert Rev Mol Med 9, 1-16 (2007).

72. Pober, B. R. Williams-Beuren syndrome. N. Engl. J. Med. 362, 239-252 (2010).

73. Tartaglia, M. et al. Mutations in PTPN11, encoding the protein tyrosine phosphatase SHP-2, cause Noonan syndrome. Nat. Genet. 29, 465-468 (2001).

74. Gamazon, E. R. et al. A gene-based association method for mapping traits using reference transcriptome data. Nat. Genet. 47, 1091-1098 (2015).

75. Gusev, A. et al. Integrative approaches for large-scale transcriptome-wide association studies. Nat Genet 48, 245-252 (2016).

76. Zhu, Z. et al. Integration of summary data from GWAS and eQTL studies predicts complex trait gene targets. Nat. Genet. 48, 481-487 (2016).

77. Finucane, H. K. et al. Heritability enrichment of specifically expressed genes identifies disease-relevant tissues and cell types. Nat Genet 50, 621-629 (2018).

78. Law, C. W., Chen, Y., Shi, W. \& Smyth, G. K. voom: precision weights unlock linear model analysis tools for RNA-seq read counts. Genome Biology 15, R29 (2014).

79. Auton, A. et al. A global reference for human genetic variation. Nature 526, 68-74 (2015).

80. Finucane, H. K. et al. Partitioning heritability by functional annotation using genome-wide association summary statistics. Nat. Genet. 47, 1228-1235 (2015).

81. Regier, A. A. et al. Functional equivalence of genome sequencing analysis pipelines enables harmonized variant calling across human genetics projects. Nature Communications 9, 4038 (2018).

82. Li, H. Aligning sequence reads, clone sequences and assembly contigs with BWA-MEM. arXiv:1303.3997 [q-bio] (2013).

83. Van der Auwera, G. A. et al. From FastQ data to high confidence variant calls: the Genome Analysis Toolkit best practices pipeline. Curr Protoc Bioinformatics 43, 11.10.1-33 (2013). 
84. McLaren, W. et al. The Ensembl Variant Effect Predictor. Genome Biology 17, 122 (2016).

85. Karczewski, K. J. et al. The mutational constraint spectrum quantified from variation in 141,456 humans. Nature 581, 434-443 (2020).

86. Therneau, T. M. \& Grambsch, P. M. Modeling Survival Data: Extending the Cox Model. (Springer-Verlag, 2000). doi:10.1007/978-1-4757-3294-8. 


\section{Tables}

Table 1: Participant characteristics

\begin{tabular}{|c|c|c|}
\hline & Women & Men \\
\hline Participants & 21,932 & 19,169 \\
\hline Age at time of MRI & $64(8)$ & $65(8)$ \\
\hline BMI $\left(\mathrm{kg} / \mathrm{m}^{\wedge} 2\right)$ & $26(5)$ & $27(4)$ \\
\hline Height $(\mathrm{cm})$ & $163(6)$ & $176(7)$ \\
\hline Weight $(\mathrm{kg})$ & $69(13)$ & $83(13)$ \\
\hline Systolic Blood Pressure $(\mathrm{mmHg})$ & $136(19)$ & $142(17)$ \\
\hline Diastolic Blood Pressure $(\mathrm{mmHg})$ & $77(10)$ & $81(10)$ \\
\hline \multicolumn{3}{|l|}{ Drinking Status } \\
\hline Current & $20122(92 \%)$ & $18001(94 \%)$ \\
\hline Never & $900(4 \%)$ & $439(2 \%)$ \\
\hline Prefer not to answer & $7(0 \%)$ & $12(0 \%)$ \\
\hline Previous & $746(3 \%)$ & $603(3 \%)$ \\
\hline Standard drinks/week & $4.7(5.3)$ & $5.6(6.8)$ \\
\hline \multicolumn{3}{|l|}{ Smoking status } \\
\hline Current & $605(3 \%)$ & $796(4 \%)$ \\
\hline Never & $14334(65 \%)$ & $11289(59 \%)$ \\
\hline Prefer not to answer & $85(0 \%)$ & $49(0 \%)$ \\
\hline Previous & $6751(31 \%)$ & $6921(36 \%)$ \\
\hline Smoking quantity (pack years) & $17(14)$ & $21(17)$ \\
\hline Right atrium maximum area $\left(\mathrm{cm}^{\wedge} 2\right)$ & $22(4)$ & $26(5)$ \\
\hline Right atrium minimum area $\left(\mathrm{cm}^{\wedge} 2\right)$ & $13(3)$ & $16(4)$ \\
\hline Right atrium fractional area change (\%) & $42(6)$ & $40(6)$ \\
\hline Right ventricular end diastolic volume $(\mathrm{mL})$ & $112(21)$ & $155(30)$ \\
\hline Right ventricular end systolic volume $(\mathrm{mL})$ & $44(11)$ & $66(17)$ \\
\hline Right ventricular stroke volume $(\mathrm{mL})$ & $68(14)$ & $89(19)$ \\
\hline Right ventricular ejection fraction (\%) & $61(6)$ & $57(6)$ \\
\hline Proximal pulmonary artery diameter $(\mathrm{cm})$ & $2.5(0.3)$ & $2.7(0.4)$ \\
\hline Pulmonary artery root diameter $(\mathrm{cm})$ & $2.5(0.3)$ & $2.9(0.3)$ \\
\hline RA maximum area, $B S A$ indexed $\left(\mathrm{cm}^{\wedge} 2 / \mathrm{m}^{\wedge} 2\right)$ & $13(2)$ & $13(2)$ \\
\hline RA minimum area, BSA indexed $\left(\mathrm{cm}^{\wedge} 2 / \mathrm{m}^{\wedge} 2\right)$ & $7.3(1.6)$ & $7.9(1.8)$ \\
\hline $\mathrm{RV}$ end diastolic volume, $\mathrm{BSA}$ indexed $\left(\mathrm{mL} / \mathrm{m}^{\wedge} 2\right)$ & $64(10)$ & $77(14)$ \\
\hline $\mathrm{RV}$ end systolic volume, $\mathrm{BSA}$ indexed $\left(\mathrm{mL} / \mathrm{m}^{\wedge} 2\right)$ & $25(6)$ & $33(8)$ \\
\hline RV stroke volume, BSA indexed $\left(\mathrm{mL} / \mathrm{m}^{\wedge} 2\right)$ & $39(7)$ & $44(9)$ \\
\hline Proximal PA diameter, BSA indexed $\left(\mathrm{cm} / \mathrm{m}^{\wedge} 2\right)$ & $1.4(0.2)$ & $1.3(0.2)$ \\
\hline
\end{tabular}




\begin{tabular}{|r|r|r|}
\hline PA root diameter, BSA indexed $\left(\mathrm{cm} / \mathrm{m}^{\wedge} 2\right)$ & $1.4(0.2)$ & $1.4(0.2)$ \\
\hline Left ventricular end diastolic volume $(\mathrm{mL})$ & $123(20)$ & $153(28)$ \\
\hline Left ventricular end systolic volume $(\mathrm{mL})$ & $41(11)$ & $58(16)$ \\
\hline Left ventricular stroke volume $(\mathrm{mL})$ & $81(12)$ & $95(16)$ \\
\hline Left ventricular ejection fraction $(\%)$ & $67(5)$ & $63(6)$ \\
\hline
\end{tabular}

Table 1 displays characteristics of the 41,101 participants whose data contributed to at least one right heart phenotype GWAS. For quantitative phenotypes, values shown represent mean (SD). For count data, values shown represent count (\%).

\section{Table 2: Lead SNPs}

\begin{tabular}{|c|c|c|c|c|c|c|c|c|c|c|}
\hline Trait & CHR & BP & SNP & $\begin{array}{l}\text { Effect } \\
\text { Allele }\end{array}$ & $\begin{array}{l}\text { Other } \\
\text { Allele }\end{array}$ & EAF & BETA & SE & P Value & $\begin{array}{l}\text { Nearest } \\
\text { Gene }\end{array}$ \\
\hline RA Max & 2 & 179362667 & rs376136055 & GT & $G$ & 0.92 & 0.074 & 0.012 & $6.50 \mathrm{E}-10$ & PLEKHA3 \\
\hline RA Max & 5 & 172664163 & rs6882776 & G & A & 0.71 & -0.041 & 0.007 & 4.60E-09 & NKX2-5 \\
\hline RA Max & 6 & 22613847 & rs7757005 & G & A & 0.64 & -0.046 & 0.007 & $2.00 \mathrm{E}-11$ & HDGFL1 \\
\hline RA Max & 12 & 4384844 & rs76895963 & $\mathrm{T}$ & $G$ & 0.98 & -0.167 & 0.025 & $3.40 \mathrm{E}-11$ & CCND2 \\
\hline RA Max & 12 & 66376091 & rs7306710 & $\mathrm{T}$ & C & 0.48 & 0.042 & 0.006 & $3.50 \mathrm{E}-11$ & HMGA2 \\
\hline RA Max & 12 & 115162091 & & $\begin{array}{l}\text { GTGTGCC } \\
\text { CC }\end{array}$ & $G$ & 0.62 & 0.039 & 0.007 & 7.60E-09 & TBX3 \\
\hline RA Max & 17 & 45280802 & rs117154502 & $\mathrm{T}$ & G & 0.94 & -0.089 & 0.013 & $2.60 \mathrm{E}-11$ & MYL4 \\
\hline RA Max & 17 & 61772449 & & GA & $G$ & 0.64 & -0.041 & 0.007 & 3.20E-09 & MAP3К3 \\
\hline RA Max & 19 & 46312077 & rs12460541 & $G$ & A & 0.65 & -0.045 & 0.007 & $9.60 \mathrm{E}-12$ & RSPH6A \\
\hline RA Min & 2 & 179649461 & rs59532220 & G & A & 0.92 & 0.095 & 0.012 & 4.10E-15 & TTN \\
\hline RA Min & 3 & 156827227 & rs11928162 & C & $\mathrm{T}$ & 0.53 & -0.037 & 0.007 & 1.30E-08 & CCNL1 \\
\hline RA Min & 5 & 138870187 & rs28883370 & C & A & 0.89 & -0.061 & 0.010 & 3.10E-09 & TMEM173 \\
\hline RA Min & 5 & 172662024 & rs2277923 & $\mathrm{T}$ & C & 0.70 & -0.053 & 0.007 & $1.00 \mathrm{E}-13$ & NKX2-5 \\
\hline RA Min & 6 & 22613847 & rs7757005 & G & A & 0.64 & -0.045 & 0.007 & 8.10E-11 & HDGFL1 \\
\hline RA Min & 6 & 32454278 & rs75219938 & C & $\mathrm{T}$ & 0.59 & 0.046 & 0.007 & 4.70E-11 & HLA-DRB5 \\
\hline RA Min & 6 & 118654308 & rs12212795 & G & C & 0.95 & 0.082 & 0.014 & 6.90E-09 & SLC35F1 \\
\hline RA Min & 8 & 32413240 & rs112852637 & $\mathrm{T}$ & C & 0.53 & -0.038 & 0.007 & 7.80E-09 & NRG1 \\
\hline RA Min & 12 & 66376091 & rs7306710 & $\mathrm{T}$ & C & 0.48 & 0.036 & 0.007 & 1.70E-08 & HMGA2 \\
\hline RA Min & 12 & 115162091 & & $\begin{array}{l}\text { GTGTGCC } \\
\text { CC }\end{array}$ & G & 0.62 & 0.043 & 0.007 & $4.80 \mathrm{E}-10$ & TBX3 \\
\hline RA Min & 17 & 45280802 & rs117154502 & $\mathrm{T}$ & $G$ & 0.94 & -0.091 & 0.014 & $2.40 \mathrm{E}-11$ & MYL4 \\
\hline RA Min & 19 & 46311420 & rs56309034 & C & $\mathrm{T}$ & 0.65 & -0.058 & 0.007 & $1.80 \mathrm{E}-17$ & RSPH6A \\
\hline RA FAC & 2 & 179649461 & rs59532220 & G & A & 0.92 & -0.079 & 0.013 & 3.30E-09 & TTN \\
\hline RA FAC & 5 & 172644017 & rs12652726 & $\mathrm{C}$ & $\mathrm{T}$ & 0.86 & 0.066 & 0.011 & $2.00 \mathrm{E}-10$ & NKX2-5 \\
\hline RA FAC & 8 & 11783118 & rs12216858 & C & $\mathrm{T}$ & 0.59 & 0.042 & 0.007 & 1.20E-08 & DEFB136 \\
\hline RA FAC & 14 & 23866713 & rs412768 & A & G & 0.69 & 0.056 & 0.008 & 7.90E-13 & MYH6 \\
\hline RA FAC & 19 & 46311420 & rs56309034 & C & $T$ & 0.65 & 0.046 & 0.008 & 1.10E-09 & RSPH6A \\
\hline RVEDV & 2 & 179753245 & rs7573293 & C & $\mathrm{T}$ & 0.28 & -0.035 & 0.006 & 2.70E-09 & CCDC141 \\
\hline RVEDV & 3 & 14260417 & rs11721007 & G & A & 0.66 & 0.033 & 0.006 & 1.10E-08 & LSM3 \\
\hline RVEDV & 3 & 99779984 & rs57848867 & A & $T$ & 0.53 & -0.034 & 0.005 & $9.00 \mathrm{E}-11$ & FILIP1L \\
\hline RVEDV & 6 & 7691688 & rs9392916 & $\mathrm{T}$ & A & 0.55 & -0.029 & 0.005 & 4.50E-08 & BMP6 \\
\hline RVEDV & 6 & 31306915 & rs28753003 & C & $\mathrm{T}$ & 0.56 & 0.036 & 0.006 & $1.60 \mathrm{E}-10$ & HLA-B \\
\hline RVEDV & 6 & 34205465 & rs202228093 & G & GGAGCCC & 0.11 & 0.050 & 0.009 & 9.90E-09 & HMGA1 \\
\hline RVEDV & 6 & 126851160 & rs1490384 & C & $\mathrm{T}$ & 0.50 & -0.032 & 0.005 & $3.70 \mathrm{E}-10$ & CENPW \\
\hline RVEDV & 6 & 130349119 & rs6569648 & C & $\mathrm{T}$ & 0.24 & 0.034 & 0.006 & 4.20E-08 & L3MBTL3 \\
\hline RVEDV & 12 & 4384844 & rs76895963 & $\mathrm{T}$ & G & 0.98 & -0.123 & 0.021 & 6.10E-09 & CCND2 \\
\hline RVEDV & 12 & 66327632 & rs10878349 & A & $G$ & 0.49 & 0.037 & 0.005 & $9.00 \mathrm{E}-13$ & HMGA2 \\
\hline RVEDV & 12 & 111884608 & rs3184504 & $\mathrm{T}$ & C & 0.47 & -0.045 & 0.005 & $4.50 \mathrm{E}-18$ & SH2B3 \\
\hline RVEDV & 13 & 50764607 & rs71190365 & C & CTT & 0.66 & -0.036 & 0.006 & $5.20 \mathrm{E}-10$ & DLEU1 \\
\hline RVEDV & 17 & 45128762 & rs1056064 & $\mathrm{T}$ & C & 0.83 & -0.047 & 0.007 & $2.40 \mathrm{E}-11$ & GOSR2 \\
\hline RVEDV & 19 & 41910617 & rs4802117 & G & A & 0.40 & 0.031 & 0.005 & 4.20E-09 & BCKDHA \\
\hline RVEDV & 20 & 32987687 & rs62212171 & $\mathrm{T}$ & C & 0.86 & 0.050 & 0.008 & $9.80 \mathrm{E}-11$ & $\mathrm{ITCH}$ \\
\hline RVESV & 1 & 228556788 & rs3738685 & C & $\mathrm{T}$ & 0.63 & -0.031 & 0.006 & 2.00E-08 & OBSCN \\
\hline RVESV & 2 & 26922062 & rs1314982 & G & A & 0.26 & 0.039 & 0.006 & $2.60 \mathrm{E}-10$ & KCNK3 \\
\hline RVESV & 2 & 179775152 & rs955738 & $\mathrm{T}$ & C & 0.52 & -0.034 & 0.005 & 4.30E-11 & CCDC141 \\
\hline
\end{tabular}


bioRxiv preprint doi: https://doi.org/10.1101/2021.02.05.429046; this version posted February 6, 2021. The copyright holder for this preprint

(which was not certified by peer review) is the author/funder. All rights reserved. No reuse allowed without permission.

\begin{tabular}{|c|c|c|c|c|c|c|c|c|c|c|}
\hline RVESV & 3 & 14260417 & rs11721007 & $G$ & A & 0.66 & 0.050 & 0.006 & $2.40 \mathrm{E}-18$ & LSM3 \\
\hline RVESV & 3 & 99779984 & rs57848867 & A & $T$ & 0.53 & -0.032 & 0.005 & 7.50E-09 & FILIP1L \\
\hline RVESV & 3 & 134444297 & rs77709005 & C & $\mathrm{T}$ & 0.85 & 0.043 & 0.008 & 1.60E-08 & EPHB1 \\
\hline RVESV & 3 & 158232862 & rs56279242 & C & $T$ & 0.56 & -0.041 & 0.005 & 7.30E-14 & RSRC1 \\
\hline RVESV & 5 & 35191701 & rs67209755 & $T$ & $\mathrm{C}$ & 0.81 & 0.038 & 0.007 & 3.90E-08 & PRLR \\
\hline RVESV & 6 & 126710804 & rs77560239 & C & CTGTG & 0.56 & -0.040 & 0.006 & $1.60 \mathrm{E}-13$ & CENPW \\
\hline RVESV & 8 & 11682267 & rs34015932 & C & $T$ & 0.79 & 0.037 & 0.007 & 4.10E-08 & FDFT1 \\
\hline RVESV & 8 & 145018354 & rs11786896 & C & $T$ & 0.95 & 0.071 & 0.013 & 1.50E-08 & PLEC \\
\hline RVESV & 10 & 121415685 & rs72840788 & $G$ & A & 0.78 & 0.060 & 0.007 & 1.10E-19 & BAG3 \\
\hline RVESV & 12 & 66327632 & rs10878349 & A & $G$ & 0.49 & 0.035 & 0.005 & $1.30 \mathrm{E}-10$ & HMGA2 \\
\hline RVESV & 12 & 111884608 & rs3184504 & $\mathrm{T}$ & $\mathrm{C}$ & 0.47 & -0.046 & 0.005 & $1.80 \mathrm{E}-17$ & $\mathrm{SH} 2 \mathrm{~B} 3$ \\
\hline RVESV & 13 & 50764607 & rs71190365 & C & CTT & 0.66 & -0.034 & 0.006 & 1.30E-08 & DLEU1 \\
\hline RVESV & 17 & 40023617 & rs781797066 & $\mathrm{T}$ & TA & 0.83 & -0.041 & 0.007 & 4.00E-08 & ACLY \\
\hline RVESV & 17 & 45013271 & rs17608766 & $T$ & C & 0.86 & -0.045 & 0.008 & $2.50 \mathrm{E}-09$ & GOSR2 \\
\hline RVESV & 19 & 41946095 & rs2231935 & C & $G$ & 0.66 & -0.032 & 0.006 & 4.30E-08 & ATP5SL \\
\hline RVESV & 19 & 46230786 & rs4803850 & $\mathrm{C}$ & $\mathrm{T}$ & 0.84 & -0.043 & 0.007 & 1.00E-08 & FBX046 \\
\hline RVESV & 20 & 33576989 & rs1885120 & $\mathrm{C}$ & G & 0.10 & -0.050 & 0.009 & 4.20E-08 & MYH7B \\
\hline RV Stroke Volume & 6 & 7708631 & rs9392918 & $\mathrm{T}$ & $\mathrm{C}$ & 0.52 & -0.038 & 0.006 & $2.40 \mathrm{E}-10$ & BMP6 \\
\hline RV Stroke Volume & 6 & 31228357 & rs6939900 & $\mathrm{C}$ & $\mathrm{T}$ & 0.64 & 0.038 & 0.006 & 2.60E-09 & HLA-C \\
\hline RV Stroke Volume & 12 & 66327632 & rs10878349 & A & $G$ & 0.49 & 0.032 & 0.006 & 4.30E-08 & HMGA2 \\
\hline RV Stroke Volume & 12 & 112059557 & rs11065979 & C & $\mathrm{T}$ & 0.57 & 0.036 & 0.006 & 2.00E-09 & BRAP \\
\hline RV Stroke Volume & 20 & 34025756 & rs143384 & A & $G$ & 0.59 & -0.038 & 0.006 & $2.80 \mathrm{E}-10$ & GDF5 \\
\hline RVEF & 1 & 16337509 & rs1763607 & $G$ & A & 0.41 & 0.042 & 0.007 & 7.30E-10 & C1orf64 \\
\hline RVEF & 2 & 179558366 & rs2042995 & $\mathrm{T}$ & $\mathrm{C}$ & 0.77 & -0.047 & 0.008 & 9.30E-09 & TTN \\
\hline RVEF & 3 & 14296182 & rs113308768 & $T$ & TTG & 0.66 & -0.054 & 0.007 & $8.40 \mathrm{E}-14$ & LSM3 \\
\hline RVEF & 3 & 158286103 & rs7613814 & C & $\mathrm{T}$ & 0.49 & 0.049 & 0.007 & $3.10 \mathrm{E}-13$ & MLF1 \\
\hline RVEF & 6 & 32609698 & rs9272739 & $\mathrm{T}$ & $\mathrm{C}$ & 0.79 & -0.052 & 0.009 & $3.90 \mathrm{E}-08$ & HLA-DQA1 \\
\hline RVEF & 8 & 11361261 & rs13275864 & C & $G$ & 0.48 & 0.049 & 0.007 & $2.40 \mathrm{E}-13$ & BLK \\
\hline RVEF & 8 & 125861374 & rs7461129 & C & $\mathrm{T}$ & 0.69 & -0.045 & 0.007 & 1.30E-09 & MTSS1 \\
\hline RVEF & 8 & 145018354 & rs11786896 & C & $T$ & 0.95 & -0.094 & 0.016 & 3.90E-09 & PLEC \\
\hline RVEF & 10 & 121429633 & rs2234962 & $\mathrm{T}$ & C & 0.78 & -0.076 & 0.008 & 8.00E-20 & BAG3 \\
\hline RVEF & 13 & 114075109 & rs76382172 & $G$ & C & 0.96 & -0.101 & 0.019 & 3.10E-08 & ADPRHL1 \\
\hline RVEF & 14 & 81171138 & rs34540535 & $\mathrm{T}$ & C & 0.96 & 0.098 & 0.017 & $2.50 \mathrm{E}-08$ & CEP128 \\
\hline RVEF & 18 & 34219777 & & G & GTT & 0.75 & -0.045 & 0.008 & 1.20E-08 & FHOD3 \\
\hline Proximal PA & 1 & 59880537 & rs12063806 & A & $\mathrm{G}$ & 0.70 & -0.053 & 0.007 & $9.60 \mathrm{E}-13$ & FGGY \\
\hline Proximal PA & 3 & 41918834 & rs71075490 & $G$ & GT & 0.16 & 0.050 & 0.009 & 3.80E-08 & ULK4 \\
\hline Proximal PA & 3 & 128202943 & rs55914222 & G & C & 0.97 & 0.115 & 0.020 & 2.90E-08 & GATA2 \\
\hline Proximal PA & 3 & 150110726 & rs13073230 & A & $\mathrm{T}$ & 0.97 & 0.111 & 0.020 & $3.50 \mathrm{E}-08$ & TSC22D2 \\
\hline Proximal PA & 3 & 169146497 & rs2014590 & C & $T$ & 0.51 & 0.046 & 0.007 & $9.60 \mathrm{E}-12$ & MECOM \\
\hline Proximal PA & 4 & 166456085 & rs6828706 & C & $T$ & 0.70 & -0.038 & 0.007 & $2.60 \mathrm{E}-08$ & CPE \\
\hline Proximal PA & 5 & 95579013 & & AT & A & 0.62 & 0.040 & 0.007 & 2.00E-09 & PCSK1 \\
\hline Proximal PA & 5 & 108090134 & rs112668446 & $\mathrm{G}$ & A & 0.92 & -0.077 & 0.013 & $2.30 \mathrm{E}-10$ & FER \\
\hline Proximal PA & 5 & 122476934 & rs335159 & C & A & 0.20 & 0.052 & 0.008 & 1.30E-10 & PRDM6 \\
\hline Proximal PA & 5 & 158261163 & rs17715065 & C & $T$ & 0.50 & 0.042 & 0.007 & $3.00 \mathrm{E}-10$ & EBF1 \\
\hline Proximal PA & 5 & 172670611 & rs35564079 & C & CT & 0.71 & -0.051 & 0.007 & $1.30 \mathrm{E}-11$ & NKX2-5 \\
\hline Proximal PA & 6 & 36646788 & rs3176320 & A & $G$ & 0.67 & -0.058 & 0.007 & $5.60 \mathrm{E}-16$ & CDKN1A \\
\hline Proximal PA & 6 & 143608968 & rs6907215 & C & $\mathrm{T}$ & 0.39 & -0.046 & 0.007 & $1.70 \mathrm{E}-11$ & AIG1 \\
\hline Proximal PA & 7 & 35273116 & rs4723399 & G & C & 0.61 & 0.056 & 0.007 & $6.40 \mathrm{E}-16$ & TBX20 \\
\hline Proximal PA & 7 & 35904160 & & GA & G & 0.98 & -0.122 & 0.022 & 3.80E-08 & SEPT7 \\
\hline Proximal PA & 7 & 73428222 & rs6974735 & A & $\mathrm{G}$ & 0.55 & -0.040 & 0.007 & 2.10E-09 & ELN \\
\hline Proximal PA & 8 & 38774696 & rs112621658 & C & A & 0.84 & 0.054 & 0.009 & 6.30E-09 & PLEKHA2 \\
\hline Proximal PA & 8 & 141057641 & rs4440615 & $G$ & A & 0.37 & -0.046 & 0.007 & $1.70 \mathrm{E}-11$ & TRAPPC9 \\
\hline Proximal PA & 10 & 79178044 & rs28735 & $G$ & $\mathrm{C}$ & 0.45 & 0.043 & 0.007 & $2.50 \mathrm{E}-10$ & KCNMA1 \\
\hline Proximal PA & 10 & 96015793 & rs3891783 & $\mathrm{C}$ & $\mathrm{G}$ & 0.56 & -0.052 & 0.007 & 1.10E-14 & PLCE1 \\
\hline Proximal PA & 11 & 17498057 & rs77889556 & $G$ & A & 0.83 & -0.070 & 0.009 & $2.30 \mathrm{E}-14$ & ABCC8 \\
\hline Proximal PA & 11 & 47370041 & rs3729989 & $T$ & C & 0.87 & -0.061 & 0.010 & $1.70 \mathrm{E}-10$ & MYBPC3 \\
\hline Proximal PA & 11 & 70040712 & & GTGGA & $G$ & 0.52 & -0.047 & 0.007 & 7.10E-12 & ANO1 \\
\hline Proximal PA & 11 & 103673277 & rs2128739 & A & C & 0.28 & 0.057 & 0.007 & $2.20 \mathrm{E}-14$ & PDGFD \\
\hline Proximal PA & 12 & 50938005 & rs573989455 & C & $\mathrm{CA}$ & 0.61 & 0.040 & 0.007 & 4.20E-08 & DIP2B \\
\hline Proximal PA & 12 & 66394664 & rs4026608 & C & $T$ & 0.39 & -0.052 & 0.007 & 1.20E-14 & HMGA2 \\
\hline Proximal PA & 12 & 71114400 & rs7304603 & $\mathrm{T}$ & $\mathrm{C}$ & 0.47 & -0.036 & 0.007 & 4.20E-08 & PTPRR \\
\hline Proximal PA & 12 & 94200555 & rs35715048 & G & A & 0.66 & 0.048 & 0.007 & $3.70 \mathrm{E}-12$ & CRADD \\
\hline Proximal PA & 13 & 50760138 & rs113539437 & $G$ & $\mathrm{C}$ & 0.53 & -0.049 & 0.007 & 3.90E-13 & DLEU1 \\
\hline Proximal PA & 15 & 48914926 & rs1036477 & A & $\mathrm{G}$ & 0.89 & -0.063 & 0.011 & 2.80E-08 & FBN1 \\
\hline
\end{tabular}


bioRxiv preprint doi: https://doi.org/10.1101/2021.02.05.429046; this version posted February $6,2021$. The copyright holder for this preprint (which was not certified by peer review) is the author/funder. All rights reserved. No reuse allowed without permission.

\begin{tabular}{|c|c|c|c|c|c|c|c|c|c|c|}
\hline Proximal PA & 15 & 57217438 & rs11465192 & G & GC & 0.48 & 0.034 & 0.007 & 1.10E-08 & TCF12 \\
\hline Proximal PA & 15 & 63895818 & rs4438258 & $\mathrm{T}$ & C & 0.88 & 0.056 & 0.010 & 3.80E-08 & FBXL22 \\
\hline Proximal PA & 16 & 70096679 & rs62052481 & C & A & 0.94 & 0.080 & 0.014 & $1.80 \mathrm{E}-08$ & PDPR \\
\hline Proximal PA & 17 & 2097483 & rs1532292 & $\mathrm{T}$ & G & 0.62 & -0.054 & 0.007 & $2.80 \mathrm{E}-15$ & SMG6 \\
\hline Proximal PA & 17 & 45138033 & rs145153053 & A & G & 0.83 & -0.059 & 0.009 & 1.90E-11 & GOSR2 \\
\hline Proximal PA & 19 & 11275411 & rs199839456 & C & $\mathrm{T}$ & 0.69 & -0.045 & 0.007 & 1.00E-09 & KANK2 \\
\hline Proximal PA & 19 & 30313576 & rs3218069 & C & $\mathrm{T}$ & 0.60 & -0.044 & 0.007 & $1.40 \mathrm{E}-10$ & CCNE1 \\
\hline Proximal PA & 19 & 39150235 & rs10415219 & A & G & 0.52 & -0.051 & 0.007 & $5.10 \mathrm{E}-14$ & ACTN4 \\
\hline PA Root & 1 & 59887078 & rs66478136 & $\mathrm{T}$ & A & 0.70 & -0.037 & 0.007 & $1.40 \mathrm{E}-08$ & FGGY \\
\hline PA Root & 1 & 100033312 & & TA & $\mathrm{T}$ & 0.49 & -0.042 & 0.006 & $7.90 \mathrm{E}-12$ & PALMD \\
\hline PA Root & 1 & 149927034 & rs12048493 & A & C & 0.61 & 0.037 & 0.006 & 2.30E-09 & OTUD7B \\
\hline PA Root & 3 & 99860401 & rs7629487 & G & A & 0.59 & 0.041 & 0.006 & $2.20 \mathrm{E}-12$ & CMSS1 \\
\hline PA Root & 4 & 115414433 & & ATAT & A & 0.62 & -0.039 & 0.006 & 2.30E-10 & UGT8 \\
\hline PA Root & 4 & 120900282 & rs13134800 & $\mathrm{T}$ & C & 0.30 & -0.047 & 0.007 & 7.30E-13 & MAD2L1 \\
\hline PA Root & 4 & 156391307 & rs17033041 & A & G & 0.80 & 0.044 & 0.008 & $9.20 \mathrm{E}-09$ & MAP9 \\
\hline PA Root & 5 & 108090134 & rs112668446 & G & A & 0.92 & -0.082 & 0.011 & $2.50 \mathrm{E}-11$ & FER \\
\hline PA Root & 5 & 122818269 & & CT & C & 0.65 & 0.037 & 0.006 & 6.70E-09 & CSNK1G3 \\
\hline PA Root & 5 & 172670611 & rs35564079 & C & CT & 0.71 & -0.047 & 0.007 & $9.50 \mathrm{E}-12$ & NKX2-5 \\
\hline PA Root & 6 & 34144189 & rs56005336 & C & $G$ & 0.96 & -0.096 & 0.015 & $8.20 \mathrm{E}-11$ & GRM4 \\
\hline PA Root & 6 & 143591821 & rs6941056 & C & $G$ & 0.56 & -0.043 & 0.006 & $1.00 \mathrm{E}-12$ & AIG1 \\
\hline PA Root & 8 & 11671041 & rs1736058 & $\mathrm{G}$ & $A$ & 0.82 & 0.045 & 0.008 & 7.30E-09 & FDFT1 \\
\hline PA Root & 8 & 109060667 & rs2514841 & C & $\mathrm{T}$ & 0.53 & 0.033 & 0.006 & $3.50 \mathrm{E}-08$ & RSPO2 \\
\hline PA Root & 10 & 30163133 & rs777393148 & TC & $\mathrm{T}$ & 0.31 & 0.037 & 0.007 & $3.20 \mathrm{E}-08$ & SVIL \\
\hline PA Root & 10 & 96038686 & rs11187838 & $\mathrm{G}$ & A & 0.56 & -0.041 & 0.006 & $6.70 \mathrm{E}-12$ & PLCE1 \\
\hline PA Root & 11 & 47380593 & rs3740689 & $G$ & A & 0.42 & 0.041 & 0.006 & $8.20 \mathrm{E}-12$ & SPI1 \\
\hline PA Root & 11 & 69825414 & rs72931748 & A & G & 0.91 & -0.056 & 0.011 & 4.70E-08 & ANO1 \\
\hline PA Root & 12 & 12883632 & & $A G$ & A & 0.75 & 0.044 & 0.008 & 1.60E-08 & APOLD1 \\
\hline PA Root & 12 & 20230639 & rs10770612 & A & G & 0.80 & -0.051 & 0.008 & $2.00 \mathrm{E}-11$ & PDE3A \\
\hline PA Root & 12 & 22005003 & rs2307024 & $\mathrm{T}$ & G & 0.59 & 0.039 & 0.006 & $1.30 \mathrm{E}-10$ & ABCC9 \\
\hline PA Root & 12 & 66394664 & rs4026608 & C & $\mathrm{T}$ & 0.39 & -0.048 & 0.006 & $3.70 \mathrm{E}-15$ & HMGA2 \\
\hline PA Root & 12 & 94130967 & rs10859568 & $\mathrm{T}$ & A & 0.55 & 0.051 & 0.006 & $6.00 \mathrm{E}-17$ & CRADD \\
\hline PA Root & 13 & 50769041 & rs796731684 & TA & $\mathrm{T}$ & 0.54 & -0.065 & 0.006 & $1.40 \mathrm{E}-26$ & DLEU1 \\
\hline PA Root & 15 & 48914926 & rs1036477 & A & G & 0.89 & -0.060 & 0.010 & 1.80E-09 & FBN1 \\
\hline PA Root & 16 & 75331064 & rs11647088 & C & G & 0.39 & 0.035 & 0.006 & $1.40 \mathrm{E}-08$ & CFDP1 \\
\hline PA Root & 16 & 88535620 & rs36049560 & A & G & 0.67 & 0.038 & 0.007 & 2.30E-09 & ZFPM1 \\
\hline PA Root & 17 & 45013271 & rs17608766 & $\mathrm{T}$ & C & 0.86 & -0.128 & 0.009 & $1.90 \mathrm{E}-51$ & GOSR2 \\
\hline PA Root & 17 & 67957712 & rs180068 & G & C & 0.74 & 0.047 & 0.007 & $1.70 \mathrm{E}-11$ & KCNJ16 \\
\hline PA Root & 19 & 39153044 & rs16972767 & G & A & 0.52 & -0.039 & 0.006 & $2.50 \mathrm{E}-10$ & ACTN4 \\
\hline PA Root & 20 & 49192312 & rs75146649 & A & $\mathrm{G}$ & 0.91 & -0.069 & 0.011 & $4.70 \mathrm{E}-12$ & PTPN1 \\
\hline
\end{tabular}

Lead SNPs from the right heart phenotypes. CHR: chromosome. BP: GRCh37 position. EAF: effect allele frequency. BETA: effect size. SE: standard error of effect size. Lead SNPs of the BSA-indexed phenotypes are listed in Supplementary Table 2. 


\section{Figures}

\section{Figure 1}

A

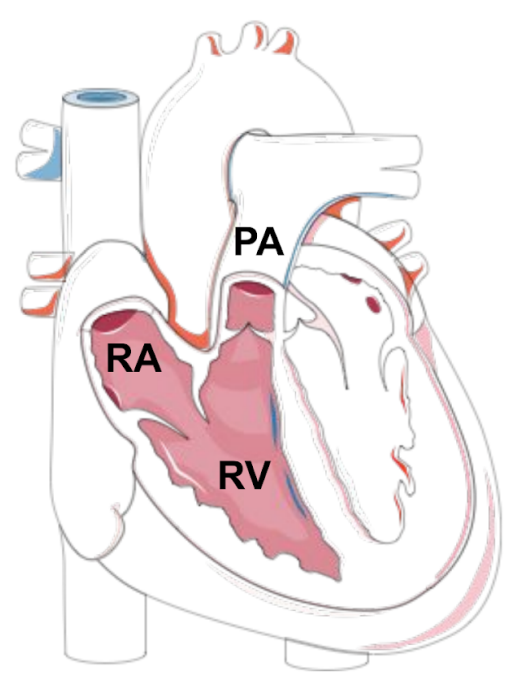

B
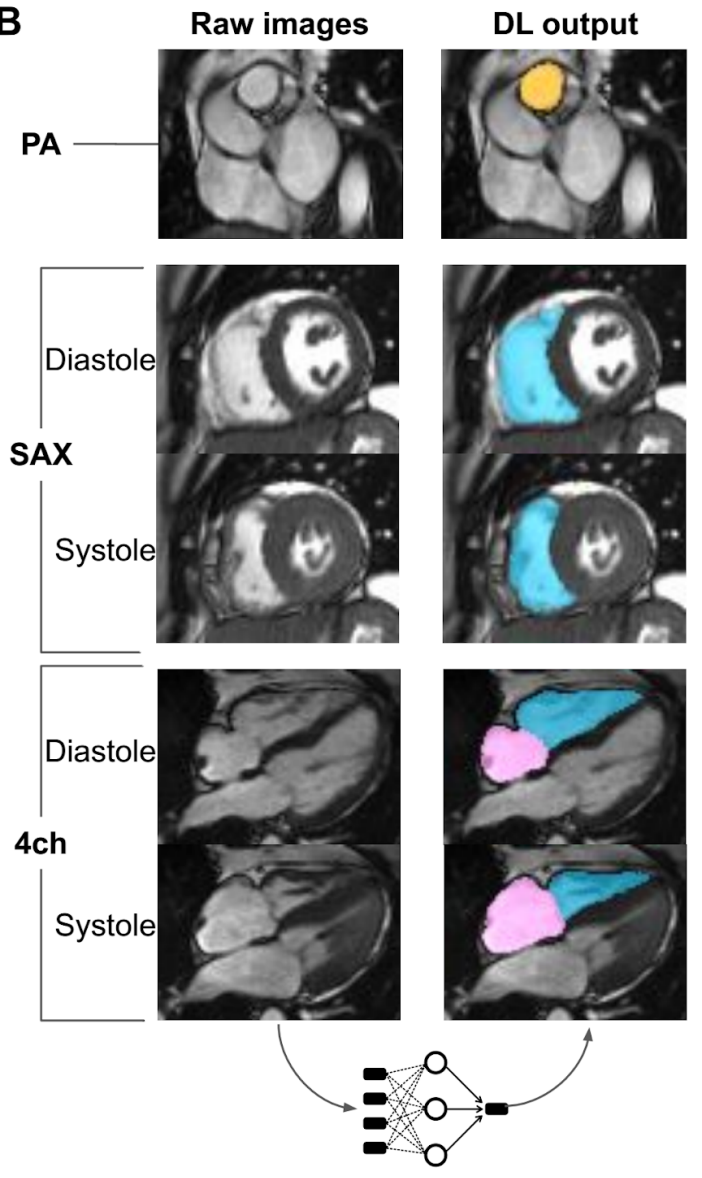

DL output
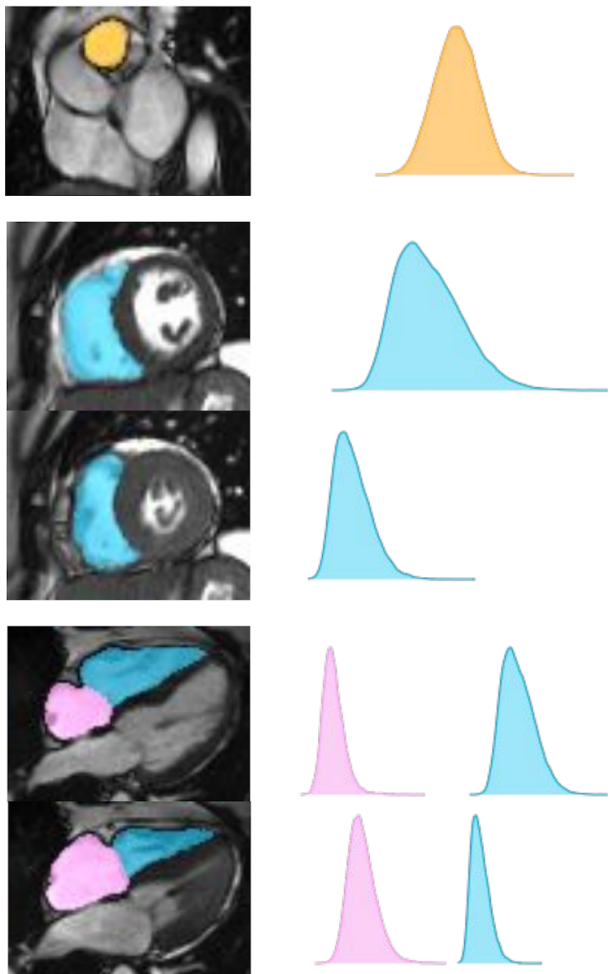

C

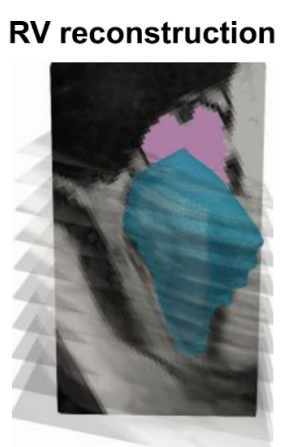

RA-RV correction

Diastole Systole

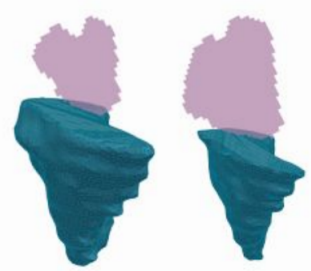

Panel A: Graphical depictions of the right heart structures in diastole and systole. RA = right atrium. $\mathrm{PA}=$ pulmonary artery. $\mathrm{RV}=$ right ventricle. In ventricular diastole, the tricuspid valve opens, allowing blood to flow from the right atrium into the right ventricle. The pulmonic valve is closed. In ventricular diastole, the right ventricle squeezes, closing the tricuspid valve and ejecting blood across the pulmonic valve into the pulmonary artery. During this time, the right 
atrium fills. The images in Panel A are derived from Servier Medical Art (licensed under creative commons by attribution). Panel B: Right heart structures and deep learning model output. PA = pulmonary artery. SAX = short axis view. $4 \mathrm{ch}=$ four-chamber long axis view. $\mathrm{DL}=$ deep learning. The pulmonary artery segmentation is colored in orange; the right ventricle is colored in blue; and the right atrium is colored in pink. The raw images on the leftmost panes are fed into the trained deep learning model, producing output that is colorized and laid on top of the raw images in the middle panes. This process is repeated for all participants and the output structures are measured, leading to population distributions of measurements as shown in the right panes. Panel C: Integration of SAX and 4ch data to reconstruct the right ventricle. The different images are aligned based on metadata provided from the MRI. A surface reconstruction technique is then applied (see Methods for details). Finally, reconstructed portions of the right ventricle that bulge into the right atrium are removed. 
bioRxiv preprint doi: https://doi.org/10.1101/2021.02.05.429046; this version posted February 6, 2021. The copyright holder for this preprint (which was not certified by peer review) is the author/funder. All rights reserved. No reuse allowed without permission.

Figure 2: Right heart structures are associated with PheCode-based disease definitions

RA Min

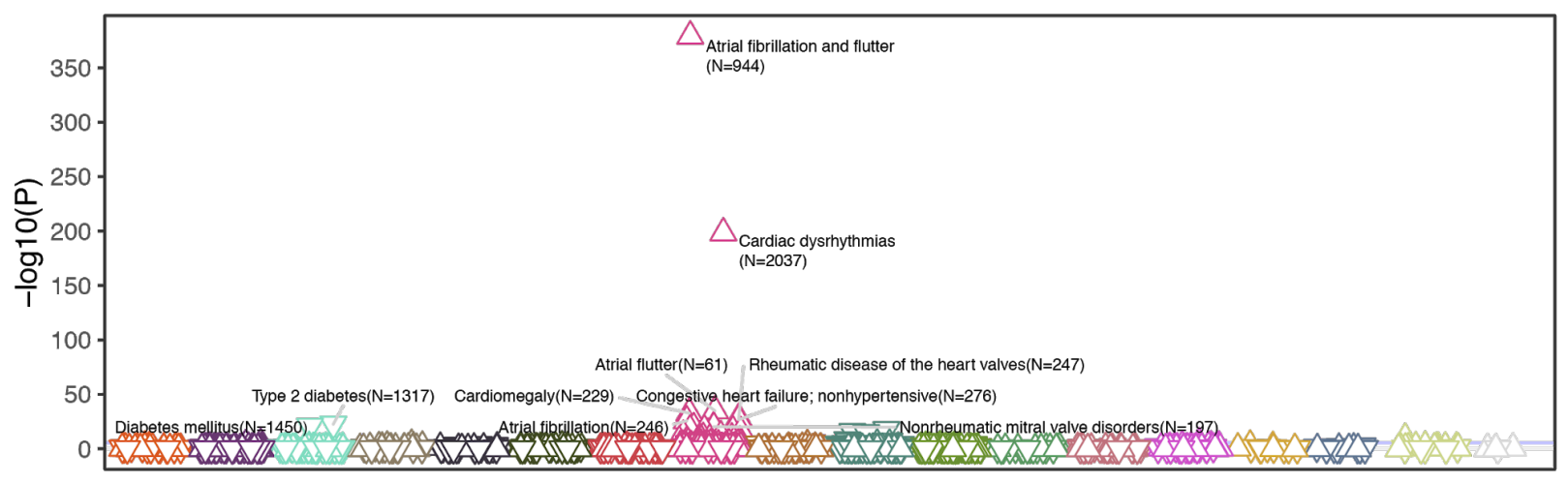

\section{RVESV}

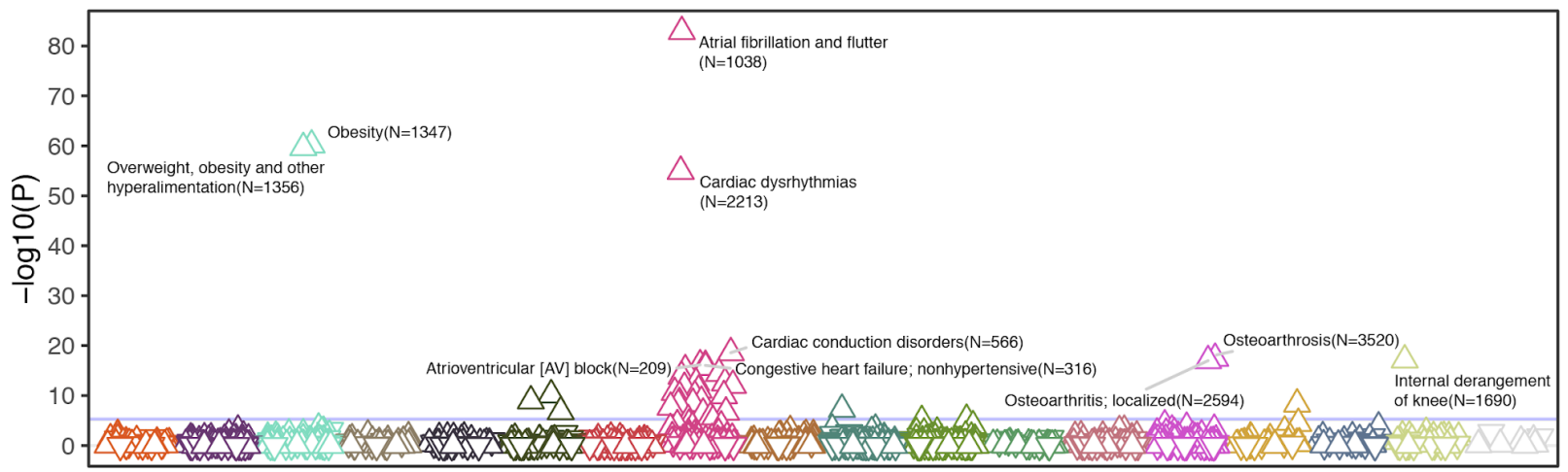

Proximal PA

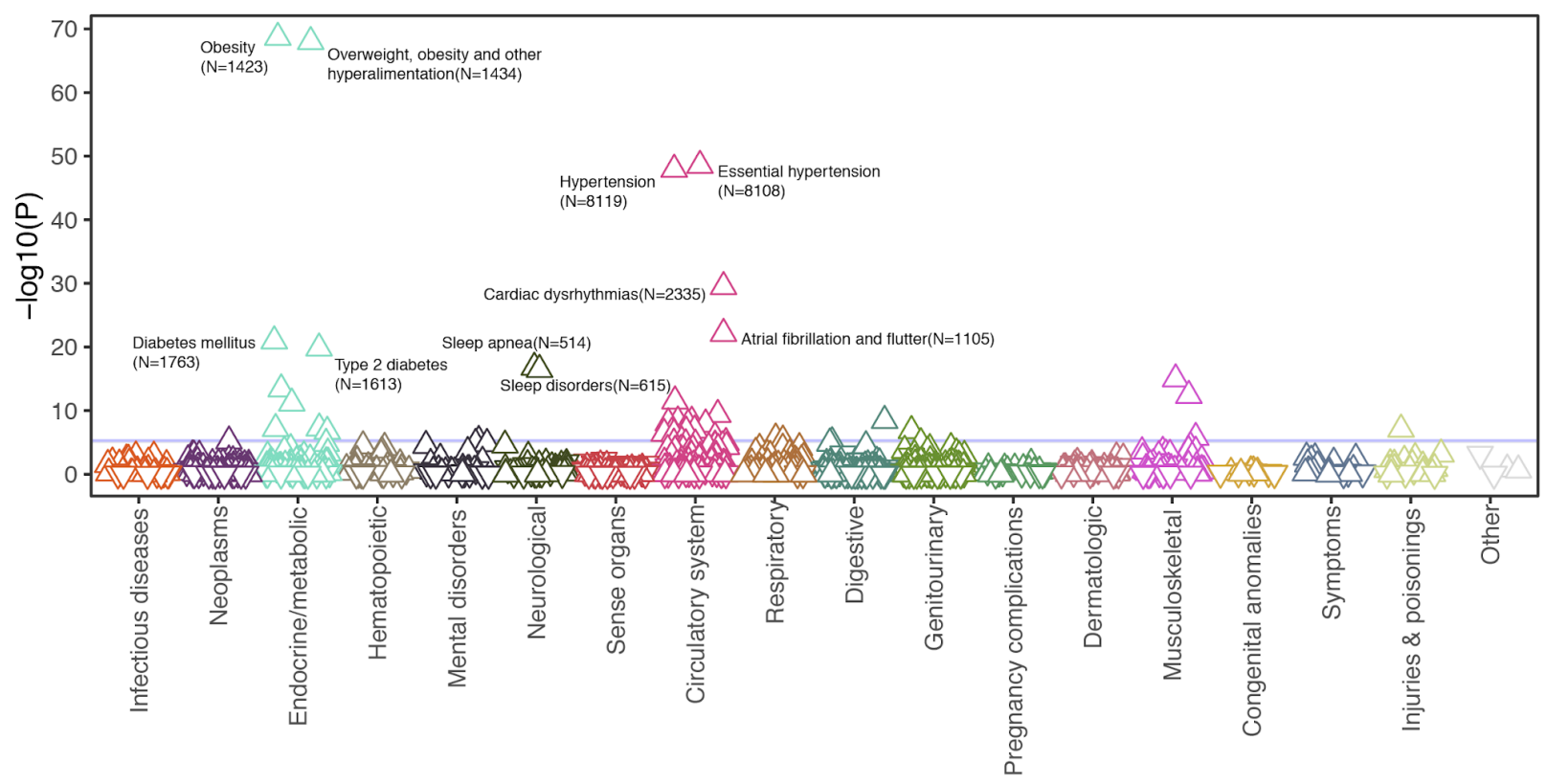


PheCode-based disease labels (X-axis) are plotted against a transformation of their association $P$ value ( $Y$-axis) with three right heart phenotypes: minimum right atrial area, right ventricular end systolic volume, and proximal pulmonary artery diameter. The modeled effect is a perturbation of the right heart trait among those with PheCode-based diseases identified prior to the time of magnetic resonance imaging, adjusting for anthropometric covariates and genetic principal components. The direction of the arrow indicates whether the presence of the disease is associated with an increase (upward arrow) or a decrease (downward arrow) of the right heart phenotype.

\section{Figure 3: Perturbations to right ventricular volumes from prevalent diseases}
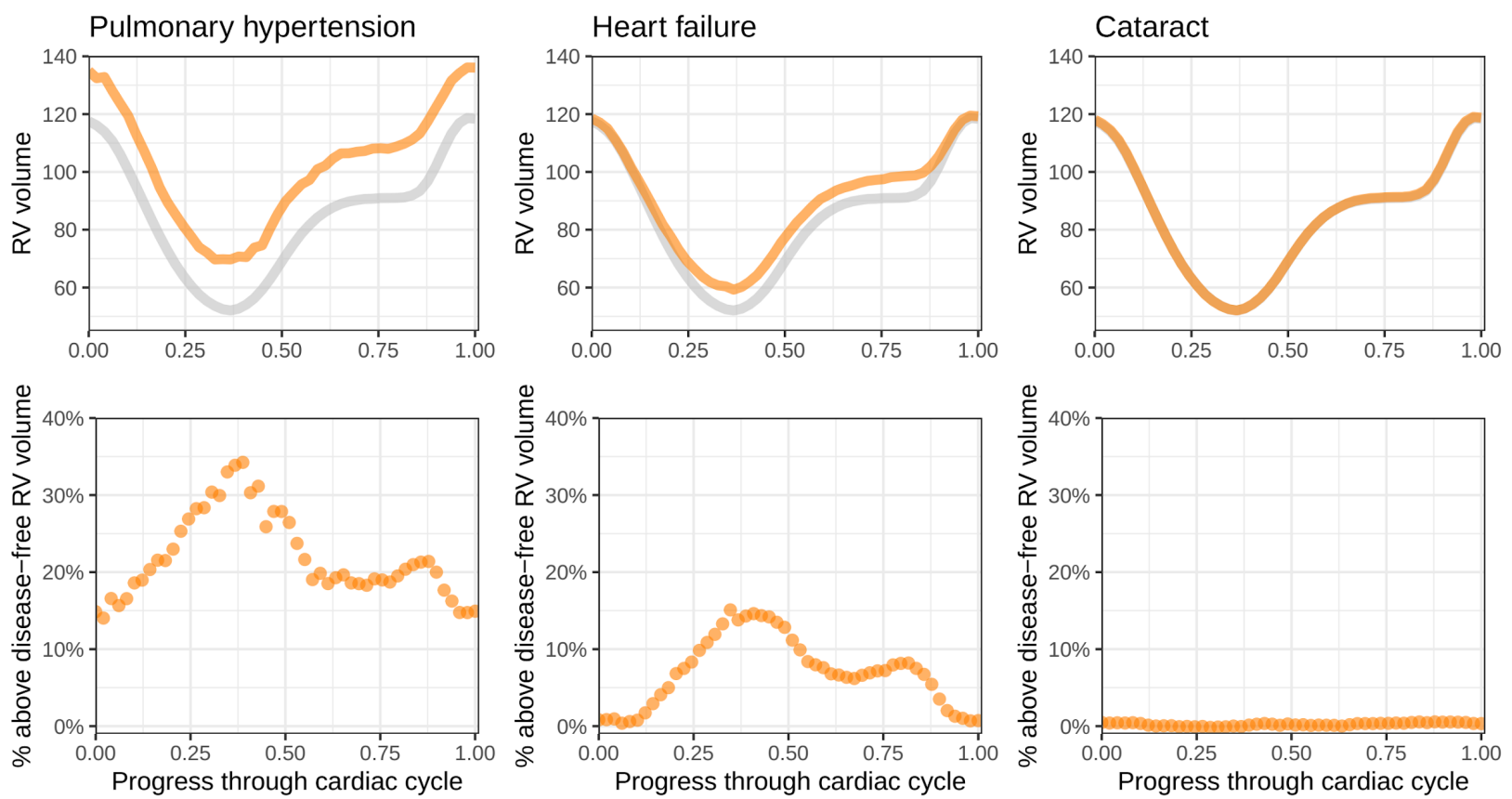

Top: Disease diagnoses that occur prior to the date of MRI are linked with distinct changes in the volume of the right ventricle throughout the cardiac cycle. The $x$-axis represents fractions of a cardiac cycle (divided evenly into 50 components, starting at end-diastole). The y-axis represents volume in $\mathrm{mL}$. Values are generated with a linear model for each time point; the gray line represents the population without disease, while the orange line represents the population with disease. In the UK Biobank, those with pulmonary hypertension have elevated RV volumes throughout the cardiac cycle, while those with heart failure predominantly have elevated endsystolic volumes. Cataract is used as a control to demonstrate little association between a noncardiovascular disease and the volume of the right ventricle. Bottom: At each time, the right ventricular volume of individuals with disease is subtracted from the volume without disease and divided by the volume without disease. This represents the percentage above or below the disease-free right ventricular volume for those with disease. 
Figure 4: Manhattan plots
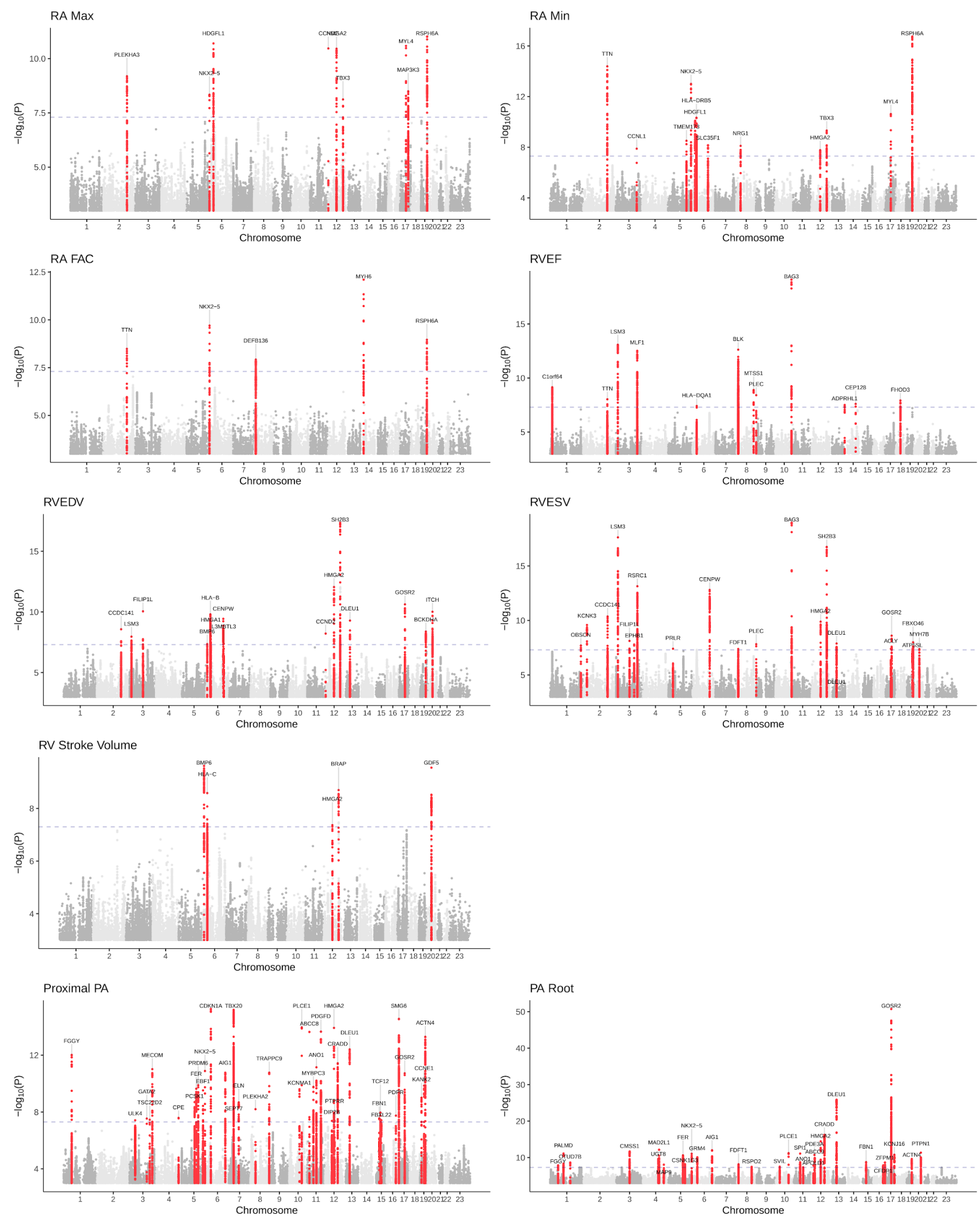
Manhattan plots show the chromosomal position (X-axis) and the strength of association ($\log 10$ of the $\mathrm{P}$ value, $\mathrm{Y}$-axis) for all non-BSA-indexed phenotypes. Loci that contain SNPs with $P<5 E-08$ are colored red and labeled with the name of the nearest gene.

Figure 5: Cumulative incidence of dilated cardiomyopathy stratified by genetic prediction of RVESV
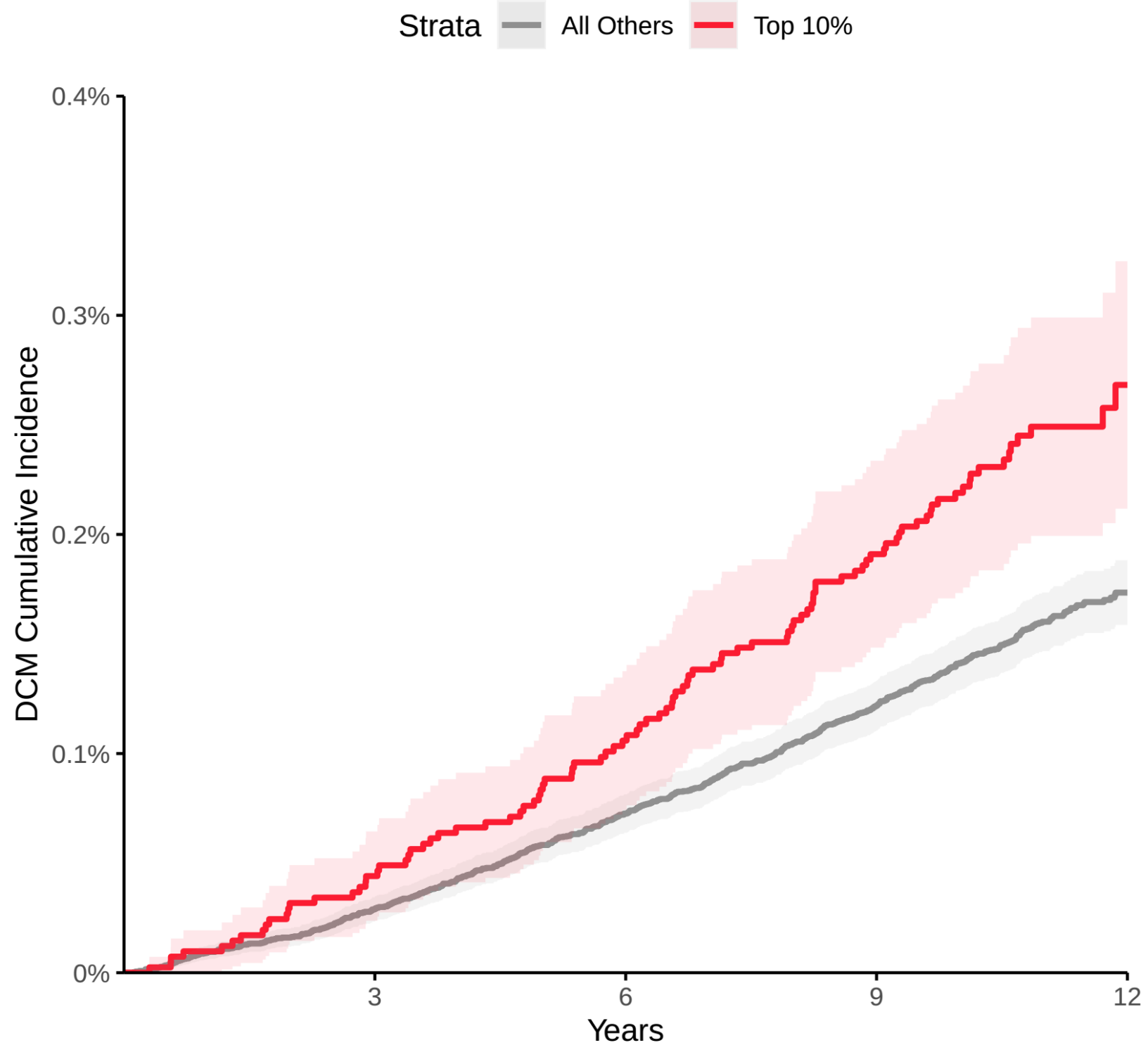

Individuals unrelated within 3 degrees of the participants who underwent MRI in the top 10\% for the RVESV PRS (red) and bottom 90\% (gray). X-axis: years since enrollment in the UK Biobank. Y-axis: cumulative incidence of dilated cardiomyopathy. Those in the top $10 \%$ of genetically predicted RVESV had an increased risk of DCM (Cox HR 1.53, P = 8E-05) compared with those in the bottom $90 \%$ in up to 12 years of follow-up time after UK Biobank enrollment. 